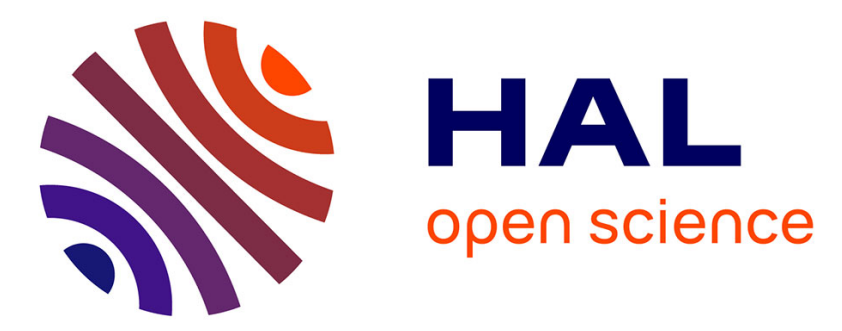

\title{
Sub-Terahertz Wireless System using Dual-Polarized Generalized Spatial Modulation with RF Impairments
}

Nizar Bouhlel, Majed Saad, Faouzi Bader

\section{To cite this version:}

Nizar Bouhlel, Majed Saad, Faouzi Bader. Sub-Terahertz Wireless System using Dual-Polarized Generalized Spatial Modulation with RF Impairments. IEEE Journal on Selected Areas in Communications, 2021, 39 (6), pp.1636-1650. 10.1109/JSAC.2021.3071828 . hal-03172151

\section{HAL Id: hal-03172151 \\ https://hal.science/hal-03172151}

Submitted on 17 Mar 2021

HAL is a multi-disciplinary open access archive for the deposit and dissemination of scientific research documents, whether they are published or not. The documents may come from teaching and research institutions in France or abroad, or from public or private research centers.
L'archive ouverte pluridisciplinaire HAL, est destinée au dépôt et à la diffusion de documents scientifiques de niveau recherche, publiés ou non, émanant des établissements d'enseignement et de recherche français ou étrangers, des laboratoires publics ou privés. 


\title{
Sub-Terahertz Wireless System using Dual-Polarized Generalized Spatial Modulation with RF Impairments
}

\author{
Nizar Bouhlel, Member, IEEE, Majed Saad, Member, IEEE, and Faouzi Bader, Senior Member, IEEE,
}

\begin{abstract}
In this paper, we address a multiple-input multipleoutput (MIMO) communication system based on generalized spatial modulation (GSM) and dual-polarized (DP) antennas in sub-TeraHertz (sub-THz) bands to improve the Spectral Efficiency (SE) while reducing the spatial correlation effect and the space occupancy. Moreover, the joint Maximum Likelihood (ML) detector and a Modified Ordered Block Minimum MeanSquared Error (MOB-MMSE) detector algorithm are proposed to detect jointly the complex symbols and the virtual bits conveyed by the activated polarization and the transmit antenna combination index. MOB-MMSE can achieve near-ML performance with low complexity. The performance of DP-GSM system over correlated Rayleigh/Rician fading and indoor subTHz channels is studied. Provided results show that DP-GSM system is robust to the spatial correlation effects under Rician fading channel. In addition, an average bit-error probability (ABEP) upper bounding is derived and analyzed for the DP-GSM system over correlated Rayleigh/Rician fading channels. Since the sub-THz band suffers from many technological limitations and severe RF-impairments such as low transmit output power and important phase noise (PN), the DP-GSM system is studied by considering the sub-THz impairments. Moreover, the Dual Polarized Spatial Multiplexing (DP-SMX) and the Uni-Polarized GSM (UP-GSM) are compared with the DP-GSM system. The results reveal that DP-GSM with low order modulation schemes as QPSK outperforms the UP-GSM. Besides, DP-GSM and DPSMX provide a good performance up to medium $\mathrm{PN}$, which is interesting for sub-THz bands, but DP-GSM can reduce the transceiver cost by using a lower number of RF chains when the used transmitter architecture is based on RF-switching.
\end{abstract}

Index Terms-Generalized Spatial Modulation (GSM), dual polarization, beyond 5G, MIMO, index modulation, SubTerahertz, spatial multiplexing, phase noise.

\section{INTRODUCTION}

$\mathbf{M}$ ULTIPLE-input multiple-output (MIMO) techniques have been widely used during the last decades and have been integrated into most of the recent digital wireless communication standards [1], because of their advantages in terms of diversity/multiplexing gain [2]. However, in order to meet the ever-increasing demands in terms of increased capacity, high data throughput, and better reliability, MIMO transceivers still need to be improved. To reach these goals, wireless ultra-high data rate needs a high system Spectral Efficiency (SE) and a large bandwidth available in the millimeter-wave $(\mathrm{mmW})$ and

Nizar Bouhlel and Majed Saad are with the IETR-CentraleSupélec lab campus of Rennes (France). Avenue de la boulaie, Rennes, France. E-mails: \{nizar.bouhlel, majed.saad\}@ centralesupelec.fr

Faouzi Bader is with the IETR-CentraleSupélec lab, campus of Rennes (France) and ISEP-Institut Supérieur d'Électronique de Paris, France. E-mail: faouzi.bader@isep.fr/faouzi.bader@supelec.fr.
sub-TeraHertz (sub-THz) bands. These bands are considered mainly for beyond $5 \mathrm{G}$ ultra-high data rate scenarios such as the close proximity peer-to-peer (P2P) applications (Kiosk downloading scenario), intra-device communication, wireless fronthaul/backhaul, and data centers [3]. Sub-THz wireless communication has emerged as a viable alternative. THz offers critical advantages and opportunities that can be harnessed for the development of advanced (sixth generation-6G) ultra-high data rate wireless systems [4]. The sub-THz band suffers from many technological limitations, and severe RF-impairments such as low output power and important phase noise [5], [6]. These RF-impairments increase at high frequencies and the signal propagation in the sub- $\mathrm{THz}$ channel suffers from high atmospheric attenuation [6]. The most important RFimpairments in the sub-THz band are the Phase Noise (PN) introduced by the local oscillators (LOs), the low output power, and the limited resolution of high-speed Analog-to-Digital Converters (ADCs).

In the context of BRAVE project [7], we explore new techniques for the wireless terabit communication system in sub-THz bands. Note that Index Modulation (IM) with advanced MIMO schemes and a huge bandwidth (around 50 $\mathrm{GHz}$ ) that can be allocated in the sub- $\mathrm{THz}$ bands (mainly between $90-200 \mathrm{GHz}$ ) [8], are the key enablers to increase the $\mathrm{SE}$ and data rates. In the last decade, different IM domains have been explored separately and jointly. The time and frequency IM domains convey additional information bits by the index of activated time slot(s) [33], and sub-carrier(s) [34], [35], respectively. Thus, these two IM domains suffer from limited SE gain and even loss compared to systems without IM and high order modulations due to discarding some available resources. However, these IM domains have an energy-efficiency advantage, which is crucial for low-power Internet-of-Things devices [36]. On the other hand, the spatial IM domain is explored, and different schemes transmitting information via the index of transmit/receive antennas appeared to target either higher energy efficiency for IoT applications (e.g., [37], [38]), or higher SE (e.g., [39]), or a balanced tradeoff between both [10]. For instance, Spatial modulation (SM) has been recognized as a low-complexity MIMO scheme [9], where a single antenna at each symbol period is activated to convey information by its index. The main advantage of SM is that the interference at the receiver and the inter-antenna synchronization at the transmitter are avoided. However, the $\mathrm{SE}$ of the SM scheme increases logarithmically with the number of transmit antennas (TAs). 
To further increase the SE, the Uni-Polarized Generalized Spatial Modulation (UP-GSM) technique was developed in [10]. It uses the spatial domain to convey information by indexing the activated transmit antenna combination (TAC) and by the $M$-ary symbols [11]. In [12], it was shown that using the UP-GSM with power-efficient single carrier modulations can successfully achieve a low power wireless Terabits system.

Despite the multiple advantages of the UP-GSM system, the latter suffers from an important performance degradation in Rician and/or spatially correlated channels. Note that the degradation due to spatial correlation is reduced in [13] but cannot be completely eliminated. In addition, the space occupancy of uni-polarized (UP) antenna arrays in MIMO systems can limit the number of antennas due to physical space limitation, especially at low-frequency bands. To deal with the space limitation, an efficient solution has been proposed for the SM-MIMO system by adding a polarization dimension to the transmitter and receiver using dual-polarized (DP) antennas that can transmit simultaneously through two orthogonal polarization directions. But in DP-SM [14], only one polarization is used to allow better separation between channels and thus better performance in highly correlated channels.

We recently proposed DP-GSM in [22] where we incorporate DP antennas with GSM to reduce the spatial correlation impact and also enhance the system SE by exploring the polarization dimension. In addition, DP-GSM allows reducing the space occupancy of antenna arrays by half without any performance degradation compared to UP-GSM. Unlike the UP-GSM, the proposed DP-GSM scheme has to detect the bits conveyed through the antenna polarization (horizontal or vertical) to the estimation of the bits spatially indexed over every active antenna, and finally, the modulated symbol bits. This paper extends the DP-GSM scheme study by considering its theoretical performance, providing a complete study in the sub-THz channels, and analyzing the robustness to RF impairments for different MIMO systems. More precisely, our main contributions are summarized as follows:

1) A novel multi-dimensional IM scheme, called DP-GSM, is proposed to convey information bits in the spatial and polarization IM domains. This proposed scheme, presented briefly in [22], allows to enhance the SE of conventional GSM [10] by $N_{a}$.

2) A joint ML detector is presented to estimate all information bits of DP-GSM in the spatial, polarization, and signal dimensions.

3) A sub-optimal detector with lower complexity named the Modified OB-MMSE (MOB-MMSE) is presented to estimate the spatial bits, the polarization bits, and the symbol bits.

4) The theoretical Average Bit-error probability (ABEP) upper bound of the DP-GSM system under the ML detector over correlated Rayleigh/Rician channels is derived in this article and verified to be tight with Monte-Carlo simulation results.

5) The performance analysis of the proposed DP-GSM system is addressed over spatially correlated Rayleigh/Rician channels with different spatial correlations and over a ray-based deterministic channel model for sub-THz Band [24]. To illustrate the potential of the proposed DPGSM system for sub-THz channels, we compared it with the UP-GSM and the dual-polarized MIMO Spatial Multiplexing (DP-SMX) systems.

6) Finally, a complete analysis is provided for the proposed DP-GSM system experiencing different levels of PN impairments and compared with the DP-SMX system.

The paper is organized as follows. In Section II, we provide a description of the DP-GSM system and the modified OBMMSE sub-optimal detector. Section III provides a detailed explanation of the theoretical general correlated channel modeling, the Ray-based deterministic channel modeling for sub$\mathrm{THz}$ bands, and the RF-impairments. Section IV details the performance analysis of the proposed DP-GSM system in terms of BER and ABEP upper bound, and it also presents the simulation results under correlated Rayleigh/Rician channels and real sub-THz channel with/without RF-impairments. Finally, the discussion and some concluding remarks close up this paper.

The notations are as follows: capital bold letters represent matrices, and bold lowercase letters are used for vectors. The term $[.]^{T}$ means the transpose operator, and the $[.]^{H}$ the Hermitian transpose. [.] ${ }^{*}$ denotes the complex conjugate of [.]. $\mathcal{R}\{$.$\} and \mathcal{I}\{$.$\} denotes the real and imaginary part of$ \{.\}. $C \mathcal{N}\left(\mu, \sigma^{2}\right)$ denotes the complex normal distribution of a random variable having mean $\mu$ and variance $\sigma^{2}$. $C_{n}^{k}$ represents the binomial coefficient indexed by a pair of integers $n \geq k \geq 0$. The delimiter $\lfloor$.」 denotes the floor function that means the largest integer less than or equal to $x$. $\|$.$\| stands$ for the Frobenius norm. The probability of an event is denoted by $P_{r}(.) . \mathbb{E}[$.$] denotes the expectation, and the operator \odot$ denotes the element-by-element Hadamard multiplication, and $\otimes$ means the Kronecker product.

\section{SYSTEM MOdEL: DUAL-POLARIZED GSM SYSTEM}

The proposed system model is a MIMO system with $N_{t}$ and $N_{r}$ DP antennas at transmitter and receiver, respectively, and based on the GSM, in which $N_{a}\left(N_{a}<N_{t}\right)$ antennas are activated in each time slot. Therefore, the number of TAC is $C_{N_{t}}^{N_{a}}$ that represents the binomial coefficient. However, only $2^{\left\lfloor\log _{2}\left(C_{N_{t}}^{N a}\right)\right\rfloor}$ TACs are permitted to keep the bits length an integer number.

The $N_{r} \times N_{t}$ DP-GSM system leads to $2 N_{r} \times 2 N_{t}$ dimensional channel between transmitter and receiver due to the implemented dual polarization. The DP-GSM system is shown in Fig. 1. At each transmission instant, a block of $L=L_{1}+L_{2}+L_{3}$ incoming data bits are selected and constituted of three parts named: $L_{1}=\left\lfloor\log _{2}\left(C_{N_{t}}^{N_{a}}\right)\right\rfloor$ bits are used to select the $N_{a}$ activated antennas at the transmitter, $L_{2}=N_{a} \log _{2} N_{p}$ bits used to select the polarizations of the selected antennas. Here $N_{p}=2$ stands the polarization dimension. Note that the polarization directions can be vertical, denoted by ' $v$ ' or horizontal denoted ' $\mathrm{h}$ '. The remaining $L_{3}=N_{a} \log _{2} M$ represent the bits mapped into $N_{a} M$-ary symbol of the transmitted constellation symbol vector $\mathbf{s}=\left[s_{1}, s_{2}, \ldots, s_{N_{a}}\right]^{\mathrm{T}}$ 


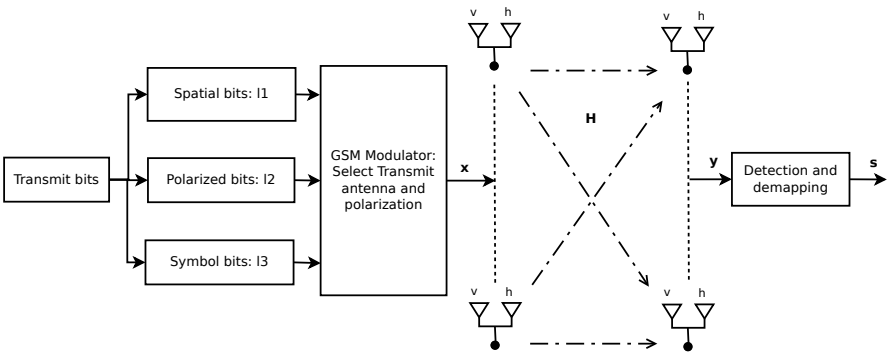

Fig. 1. $N_{r} \times N_{t}$ DP-GSM system model.

where $[.]^{\mathrm{T}}$ is the transpose operator. Thus, the total achieved $\mathrm{SE}$ in bit per channel use (bpcu) can be expressed as

$$
L=\left\lfloor\log _{2}\left(C_{N_{t}}^{N_{a}}\right)\right\rfloor+N_{a} \log _{2} M+N_{a} .
$$

By doing so, the number of possible TACs is $N_{1}=2^{\left\lfloor\log _{2}\left(C_{N_{t}}^{N a}\right)\right\rfloor}$ and the number of possible transmit polarization combinations (TPCs) is $N_{2}=2^{N a}$. Thus, the total number of possible TA and polarization combination (TAPC) is $N=N_{1} N_{2}$.

The transmitted signal is denoted by $\mathbf{x}$ and represented by a $2 N_{t} \times 1$ vector whose entries have $N_{a}$ non-zero elements. The position of the non-zero elements in $\mathbf{x}$ allows the identification of the selected TAs as well as their polarizations and the transmitted symbols. We denote in this work $i_{1}, i_{2}, \ldots, i_{N_{a}}$ as the indices of $N_{a}$ active antenna in the $i$-th TAC, where $i \in\left\{1,2, \ldots, N_{1}\right\}$ and we denote $\ell_{1}, \ell_{2}, \ldots, \ell_{N_{a}}$ as the corresponding polarization of the activated antennas at the $\ell$-th TPC where $\ell \in\left\{1,2, \ldots, N_{2}\right\}$. The TA with index $i_{q} \in\left\{1,2, \ldots, N_{t}\right\}$ and polarization $\ell_{q} \in\{\mathrm{v}, \mathrm{h}\}$ sends out symbols $s_{q}$ where $q \in\left\{1,2, \ldots, N_{a}\right\}$. The transmit signal vector $\mathbf{x}$ is given by

$$
\begin{aligned}
& \mathbf{x}=\left[\ldots,[0,0],\left[0, s_{1}\right], \ldots,\left[s_{2}, 0\right], \ldots,\left[0, s_{N_{a}}\right], \ldots\right]^{\mathrm{T}} . \\
& \begin{array}{ccc}
\downarrow & \downarrow & \downarrow \\
\ell_{1}=\mathrm{h} & \ell_{2}=\mathrm{v} & i_{N a} \\
i_{N a}=\mathrm{h}
\end{array}
\end{aligned}
$$

At receiver, $N_{r}$ DP antennas receive the modulated signal transmitted over a slow fading and frequency-flat MIMO channel. The channel matrix $\mathbf{H}$ is equivalent to a $2 N_{r} \times 2 N_{t}$ matrix represented as

$$
\mathbf{H}=\left[\begin{array}{ccccc}
h_{1 \mathrm{v}, 1 \mathrm{v}} & h_{1 \mathrm{v}, 1 \mathrm{~h}} & h_{1 \mathrm{v}, 2 \mathrm{v}} & \cdots & h_{1 \mathrm{v}, N_{t} \mathrm{~h}} \\
h_{1 \mathrm{~h}, 1 \mathrm{v}} & h_{1 \mathrm{~h}, 1 \mathrm{~h}} & h_{1 \mathrm{~h}, 2 \mathrm{v}} & \cdots & h_{1 \mathrm{~h}, N_{t} \mathrm{~h}} \\
h_{2 \mathrm{v}, 1 \mathrm{v}} & h_{2 \mathrm{v}, 1 \mathrm{~h}} & h_{2 \mathrm{v}, 2 \mathrm{v}} & \cdots & h_{2 \mathrm{v}, N_{t} \mathrm{~h}} \\
\vdots & \vdots & \vdots & \vdots & \vdots \\
h_{N_{r} \mathrm{~h}, 1 \mathrm{v}} & h_{N_{r} \mathrm{~h}, 1 \mathrm{~h}} & h_{N_{r} \mathrm{~h}, 2 \mathrm{v}} & \cdots & h_{N_{r} \mathrm{~h}, N_{t} \mathrm{~h}}
\end{array}\right],
$$

where each $h_{i^{\prime} u, j^{\prime} u^{\prime}}$ and $u, u^{\prime} \in\{\mathrm{v}, \mathrm{h}\}$, represents the fading coefficient between polarization $u$ of $i^{\prime}$-th receive antenna (RA) and the polarization $u^{\prime}$ of $j^{\prime}$-th TA. If $u^{\prime}=u, h_{i^{\prime} u, j^{\prime} u^{\prime}}$ represents the co-polar channel coefficient; otherwise, it represents the cross-polar channel coefficient. We can reformulate (3) as $\mathbf{H}=\left[\mathbf{H}_{1}, \mathbf{H}_{2}, \ldots, \mathbf{H}_{N_{t}}\right]$ where $\mathbf{H}_{j^{\prime}}=\left[\mathbf{h}_{j^{\prime} \mathrm{v}}, \mathbf{h}_{j^{\prime} \mathbf{h}}\right] \in$ $\mathbb{C}^{2 N_{r} \times 2}$ and $\mathbf{h}_{j^{\prime} \mathrm{v}}=\left[h_{1 \mathrm{v}, j^{\prime} \mathrm{v}}, h_{1 \mathrm{~h}, j^{\prime} \mathrm{v}}, h_{2 \mathrm{v}, j^{\prime} \mathrm{v}}, \ldots, h_{N_{r} \mathrm{~h}, j^{\prime} \mathrm{v}}\right]^{\mathrm{T}}$ is the a $2 N_{r} \times 1$ channel matrix for polarization $\mathrm{v}$ of $j^{\prime}$-th TA. Similarly, $\mathbf{h}_{j^{\prime} \mathrm{h}}=\left[h_{1 \mathrm{v}, j^{\prime} \mathrm{h}}, h_{1 \mathrm{~h}, j^{\prime} \mathrm{h}}, h_{2 \mathrm{v}, j^{\prime} \mathrm{h}}, \ldots, h_{N_{r} \mathrm{~h}, j^{\prime} \mathrm{h}}\right]^{\mathrm{T}}$ is the a $2 N_{r} \times 1$ channel matrix for polarization $\mathrm{h}$ of the $j^{\prime}$-th TA. To be able to model correctly the channel $\mathbf{H}$, two kind of polarizations are investigated in our paper. Correlations induced by polarization effects within each antenna, and the limited spacing between antennas [14]. These effects will be studied in the next section. The received signal $\mathbf{y} \in \mathbb{C}^{2 N_{r} \times 1}$ can be formulated as

$$
\mathbf{y}=\mathbf{H} \mathbf{x}+\mathbf{n}=\sum_{q=1}^{N_{a}} \mathbf{h}_{i_{q} \ell_{q}} s_{q}+\mathbf{n}=\mathbf{H}_{I, P} \mathbf{S}+\mathbf{n}
$$

where $\mathbf{n} \in \mathbb{C}^{2 N_{r} \times 1}$ is an additive white complex Gaussian noise vector with zero mean and variance $\sigma^{2}$ for each entry, and $\mathbf{H}_{I, P}=\left[\mathbf{h}_{i_{1} \ell_{1}}, \mathbf{h}_{i_{2} \ell_{2}}, \ldots, \mathbf{h}_{i_{N a} \ell_{N a}}\right]$ is the sub-matrix with $N_{a}$ columns of $\mathbf{H}$, corresponding to the TAC set $I$ and TPC set $P$. We recall that $\mathbf{x}$ is power normalized to have unity power.

\section{A. Joint-ML Detector}

The receiver estimates the activated antennas, their polarizations and the transmitted symbols by using joint ML detection which performs exhaustive search over all the possible transmitted signal vectors and is given by

$$
\hat{\mathbf{x}}=\underset{\mathbf{x} \in X}{\arg \min }\|\mathbf{y}-\mathbf{H x}\|^{2}
$$

where $\chi$ denotes the set of all possible transmit vectors in spatial, polarization and signal constellation domains, and $\hat{\mathbf{x}}$ is the estimated transmit vector. Based on (4), $\hat{\mathbf{x}}$ can be written as

$$
(\hat{I}, \hat{P}, \hat{\mathbf{s}})=\underset{I \in \mathbb{I}, P \in \mathbb{P}, \mathbf{s} \in \mathbb{Q}}{\arg \min }\left\|\mathbf{y}-\mathbf{H}_{I, P} \mathbf{s}\right\|^{2}
$$

where $\mathbb{I}=\left\{I_{1}, I_{2}, \ldots, I_{N_{1}}\right\}, I_{i}$ is defined as the set of the $N_{a}$ active TAs in the $i$-th TAC, $\mathbb{P}=\left\{P_{1}, P_{2}, \ldots, P_{N_{2}}\right\}, P_{\ell}$ is defined as the set of the $N_{a}$ active polarization in the $\ell$-th TPC, and $\mathbb{Q}$ is the set of $N_{a}$-dimensional modulation symbol vectors.

\section{B. Modified OB-MMSE Detector}

In order to reduce complexity by avoiding the use of the joint ML detector, we propose a modified version of OBMMSE detector which has been successfully used for UPGSM where polarization dimension is not included [15]. Therefore, in addition to the estimation of the indices of the $N_{a}$ possible activated TAs, we propose to estimate also the activated polarization. Consequently, the received signal $\mathbf{y}$ is equalized by the pseudo-inverse of each channel column to estimate $z_{i_{q} \ell_{q}}$, and then the weighting factors $w^{i, \ell}$ are calculated to measure the joint reliability of (TAC, TPC) as follows:

$$
w^{i, \ell}=\sum_{q=1}^{N_{a}}\left|z_{i_{q} \ell_{q}}\right|^{2} \quad \text { with } z_{i_{q} \ell_{q}}=\frac{\mathbf{h}_{i_{q} \ell_{q}}^{H} \mathbf{y}}{\mathbf{h}_{i_{q} \ell_{q}}^{H} \mathbf{h}_{i_{q} \ell_{q}}} .
$$

We recall the reader that the total number of TA and polarization combination $(i, \ell)$ corresponds to $N$. Sorting the weighing factor values of the vector $\mathbf{w}=\left[w^{i, \ell} ; i \in\left\{1,2, \ldots, N_{1}\right\}, \ell \in\right.$ $\left.\left\{1,2, \ldots, N_{2}\right\}\right]^{\mathrm{T}}$ in descending order, we obtain the ordered TAPC as follows

$$
\left[k_{1}, k_{2}, \ldots, k_{N}\right]=\arg \operatorname{sort}(\mathbf{w}) .
$$




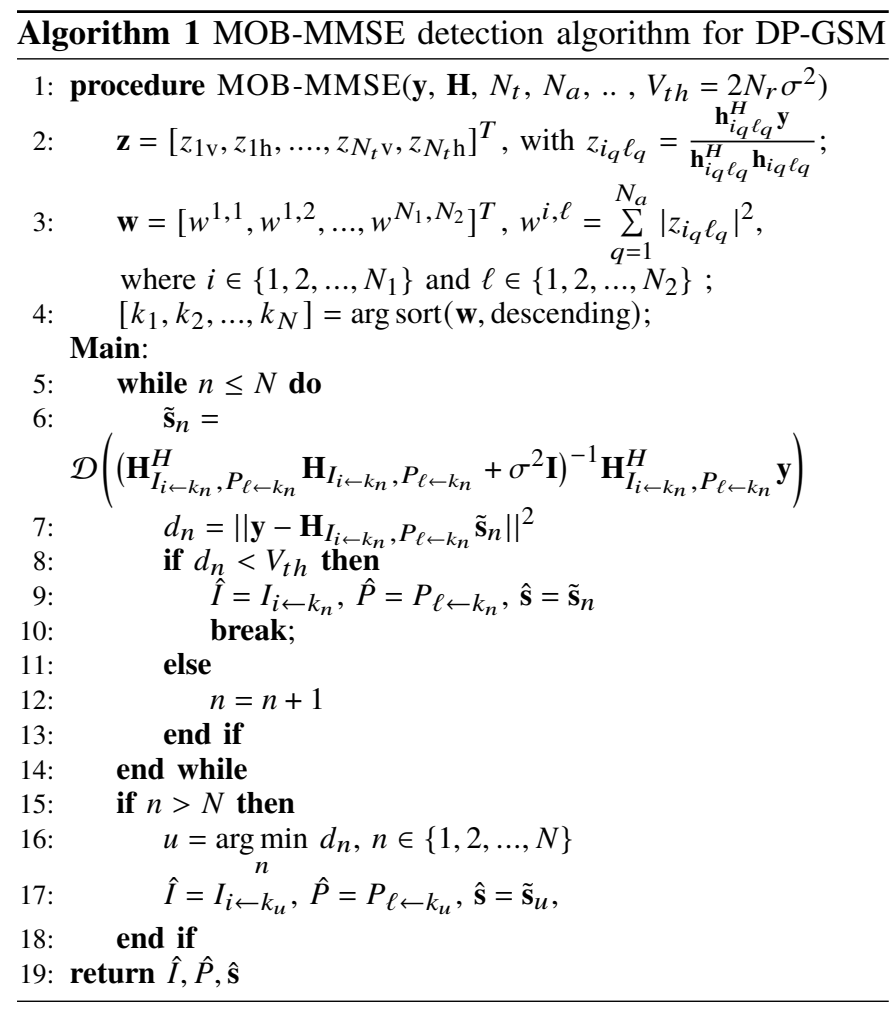

where $k_{1}$ and $k_{N}$ are the indices of the maximum and minimum value in $\mathbf{w}$, respectively. Note that the TAPC index $k_{n}$ contains implicitly the TAC and TPC indices $(i, \ell)$ and it is represented by $I_{i \leftarrow k_{n}}, P_{\ell \leftarrow k_{n}}$. Consequently, a block MMSE detector with dimension of $2 N_{r} \times N_{a}$ is used for each possible TAPC to detect the vector symbol $\tilde{\mathbf{s}}_{n}$ with $n \in\{1,2, \ldots, N\}$

$$
\tilde{\mathbf{s}}_{n}=\mathcal{D}\left(\left(\mathbf{H}_{I_{i \leftarrow k_{n}}}^{H}, P_{\ell \leftarrow k_{n}} \mathbf{H}_{I_{i \leftarrow k_{n}}, P_{\ell \leftarrow k_{n}}}+\sigma^{2} \mathbf{I}\right)^{-1} \mathbf{H}_{I_{i \leftarrow k_{n}}^{H}, P_{\ell \leftarrow k_{n}}}^{H} \mathbf{y}\right)
$$

where $\mathbf{I}$ is the $N_{a} \times N_{a}$ identity matrix and $\mathcal{D}($.$) is the$ digital demodulator function. For the reduction of the detection complexity of all $N$ possible TAPC, the MOB-MMSE will end once the output $\left(i, \ell, \tilde{\mathbf{s}}_{n}\right)$ satisfies the following relation (see the process to line 10 - Algorithm 1)

$$
\left\|\mathbf{y}-\mathbf{H}_{I_{i \leftarrow k_{n}}, P_{\ell \leftarrow k_{n}}} \tilde{\mathbf{s}}_{n}\right\|^{2} \leq V_{t h}
$$

where $V_{t h}=2 N_{r} \sigma^{2}$ is a predefined threshold used for early detection decision to reduce more the complexity. For clarification, when the transmitted DP-GSM vector (APM symbols and activated TAPC) is correctly estimated, the difference in (10) becomes: $\left\|\mathbf{y}-\mathbf{H}_{I_{i \leftarrow k n}, P_{\ell \leftarrow k n}} \tilde{\mathbf{s}}_{n}\right\|^{2}=\|\mathbf{n}\|^{2}$ that contains only the noise vector of $2 N_{r}$ elements with variance $\sigma^{2}$. Thus, the suitable threshold is $2 N_{r} \sigma^{2}$ according to [16]. If the $n$-th output satisfies the condition (10), the detector will provide the optimal TAC $\hat{I}$ and TPC $\hat{P}$, and the estimated symbol vector $\tilde{\mathbf{s}}_{n}$. Otherwise, the detector will continue the same procedure with $n=n+1$ until $n>N$. In this case, the detector becomes equivalent to the optimal ML algorithm as follows (See steps (15-18) in the Algorithm 1)

$$
\left\{\begin{array}{l}
u=\underset{n}{\arg \min } d_{n}, n \in\{1,2, \ldots, N\} \\
\hat{I}=I_{i \leftarrow k_{u}}, \hat{P}=P_{\ell \leftarrow k_{u}}, \hat{\mathbf{s}}=\tilde{\mathbf{s}}_{u} .
\end{array}\right.
$$

In the following, the computational complexity of the proposed MOB-MMSE detector is studied and compared with that of the ML detector. Note that this comparison is based on considering the number of real-valued multiplications related to the above detectors. A division operation is considered to have the same complexity as a multiplication. However, additions and subtractions are not considered in the analysis. Table I shows the achieved computational complexity using the MOB-MMSE and the ML detectors. Appendix C, provides the details of the computational complexity calculation related to these detectors. It can be noticed from Table I, that achieved computational complexity with the proposed MOB-MMSE detector is reduced compared to the ML detector. It is worth highlighting that the ML complexity increases exponentially with the size of the used constellation $\left(M^{N_{a}}\right)$ order whereas, with the MOB-MMSE detector, it increases linearly with $M$.

TABLE I

COMPARISON OF ML AND MOB-MMSE DETECTORS CONCEIVED FOR DP-GSM SYSTEM.

\begin{tabular}{|c|c|}
\hline Detectors & Computational complexity \\
\hline \hline ML & $\left(8 N_{a} N_{r}+4 N_{r}\right) 2^{L}$ \\
\hline MOB-MMSE & $\left(2 N_{a}^{3}+N_{a}^{2}\left(8 N_{r}+10\right)+N_{a}\left(16 N_{r}+2 M\right)+4 N_{r}\right) \bar{N}$ \\
& $+\left(24 N_{r} N_{t}+8 N_{t}\right)$ \\
\hline
\end{tabular}

where $\bar{N} \leq N$ is the average number of iterations executed in MOB-MMSE algorithm to detect the TAPC and APM symbols, and $L=L_{1}+L_{2}+L_{3}$.

Example: For $N_{t}=5, N_{r}=3$ and $N_{a}=3$, the complexity saving in the worst case $\bar{N}=N$ is $89.83 \%$ for $M=4$, and $99.996 \%$ for $M=64$.

\section{CHANNEL MODELS}

\section{A. General channel model}

The DP-GSM system may experience spatial correlation at transmitter and receiver due to the insufficient spacing among antennas and can be modeled as a matrix form, $\boldsymbol{\Sigma}_{t}$ and $\boldsymbol{\Sigma}_{r}$, respectively. Various models are used to describe the spatial correlations, and the exponential correlation model is used in this study [17]. Therefore, the transmit and receive spatial correlations among $i^{\prime}$-th and $j^{\prime}$-th transmit and RAs are affected, respectively, by the correlation factors $\left|\beta_{t}\right|<1$ and $\left|\beta_{r}\right|<1$ [17], where

$$
\begin{aligned}
& \left|\boldsymbol{\Sigma}_{t}\right|_{i^{\prime}, j^{\prime}}=\beta_{t}^{\left|i^{\prime}-j^{\prime}\right|}, \quad i^{\prime}, j^{\prime}=1,2, \ldots, N_{t} \\
& \left|\boldsymbol{\Sigma}_{r}\right|_{i^{\prime}, j^{\prime}}=\beta_{r}^{\left|i^{\prime}-j^{\prime}\right|}, \quad i^{\prime}, j^{\prime}=1,2, \ldots, N_{r} .
\end{aligned}
$$

In our study, the correlation matrix for the receive component is chosen equal to matrix identity, that is $\boldsymbol{\Sigma}_{r}=\mathbf{I}_{N_{r}}$ and $\beta_{t}=\beta$. The reason is to focus on the impact of correlation only at the transmitter side where a larger antenna array is used to convey the data. A low value of $\beta$ means a low spatial correlation, while larger values close to 1 mean strong spatial correlations.

The DP-GSM system may suffer also from the cross polarization correlation (XPC) between orthogonal polarization 
directions as shown in [18]. The polarization correlation at the transmitter and the receiver can be expressed as follows

$$
\boldsymbol{\Pi}_{t}=\left[\begin{array}{cc}
1 & \gamma_{t} \\
\gamma_{t}^{*} & 1
\end{array}\right], \quad \boldsymbol{\Pi}_{r}=\left[\begin{array}{cc}
1 & \gamma_{r} \\
\gamma_{r}^{*} & 1
\end{array}\right],
$$

respectively, with

$$
\begin{gathered}
\gamma_{t}=\frac{E\left\{h_{i^{\prime} \mathrm{v}, i^{\prime} \mathrm{v}} h_{i^{\prime} \mathrm{v}, i^{\prime} \mathrm{h}}^{*}\right\}}{\sqrt{\mu(1-\mu)}}=\frac{E\left\{h_{i^{\prime} \mathrm{h}, i^{\prime} \mathrm{v}} h_{i^{\prime} \mathrm{h}, i^{\prime} \mathrm{h}}^{*}\right\}}{\sqrt{\mu(1-\mu)}}, \\
\gamma_{r}=\frac{E\left\{h_{j^{\prime} \mathrm{v}, j^{\prime} \mathrm{v}} h_{j^{\prime} \mathrm{h}, j^{\prime} \mathrm{v}}^{*}\right\}}{\sqrt{\mu(1-\mu)}}=\frac{E\left\{h_{j^{\prime} \mathrm{v}, j^{\prime} \mathrm{h} h} h_{j^{\prime} \mathrm{h}, j^{\prime} \mathrm{h}}^{*}\right\}}{\sqrt{\mu(1-\mu)}}
\end{gathered}
$$

where the parameter $0<\mu \leq 1$ is the amount of power leakage from one polarization to the other [19]. Assuming the symmetry condition between different polarization, the leakage parameter is given as follows $\mu=E\left\{\left|h_{i^{\prime} \mathrm{v}, i^{\prime} \mathrm{h}}\right|^{2}\right\}=E\left\{\left|h_{i^{\prime} \mathrm{h}, i^{\prime} \mathrm{v}}\right|^{2}\right\}$ and $1-\mu=E\left\{\left|h_{i^{\prime} \mathrm{v}, i^{\prime} \mathrm{v}}\right|^{2}\right\}=E\left\{\left|h_{i^{\prime} \mathrm{h}, i^{\prime} \mathrm{h}}\right|^{2}\right\}$. In the literature, the cross-polarization discrimination (XPD) is defined as the ratio between $1-\mu$ and $\mu$. For larger XPD values, the two polarization directions are easier to be discriminated. In [20], it has been demonstrated that the impact of the polarization correlations are relatively small, $0 \leq\left|\gamma_{r}\right|,\left|\gamma_{t}\right| \leq 0.2$ and are often ignored in the derivations [14]. However, to consider better a realistic scenario in this study, we included in the model the cross-polarization correlation and power leakage effect that can occur in real hardware due to imperfect polarization isolation.

In our study, we consider a slow fading MIMO channel matrix as Rayleigh or Rician multipath fading channel with/without spatial correlation and with polarization correlation. Therefore, the $2 N_{r} \times 2 N_{t}$ DP-polarized channel matrix is expressed in the Kronecker form given by:

$$
\begin{array}{r}
\mathbf{H}=\sqrt{\frac{K}{1+K}}\left(\mathbf{1}_{N_{r} \times N_{t}} \otimes \boldsymbol{\Gamma}\right) \odot \mathbf{H}_{\mathrm{LOS}^{+}} \\
\sqrt{\frac{1}{1+K}}\left(\mathbf{1}_{N_{r} \times N_{t}} \otimes \boldsymbol{\Gamma}\right) \odot\left(\boldsymbol{\Psi}_{r}^{\frac{1}{2}} \mathbf{H}_{\mathrm{NLOS}} \boldsymbol{\Psi}_{t}^{\frac{1}{2}}\right),
\end{array}
$$

where $\boldsymbol{\Gamma}$ is the leakage matrix:

$$
\boldsymbol{\Gamma}=\left[\begin{array}{cc}
\sqrt{1-\mu} & \sqrt{\mu} \\
\sqrt{\mu} & \sqrt{1-\mu}
\end{array}\right]
$$

$\mathbf{1}_{N_{r} \times N_{t}}$ is an all one $N_{r} \times N_{t}$ matrix, $K$ is the Rician factor, $\mathbf{H}_{\mathrm{LOS}}$ and $\mathbf{H}_{\mathrm{NLOS}}$ are the $2 N_{r} \times 2 N_{t}$ line of sight (LOS) and the non LOS channel matrix respectively. The elements of $\mathbf{H}_{\mathrm{LOS}}$ are deterministic and we will assume that the elements are all one, and $\mathbf{H}_{\mathrm{NLOS}}$ is considered as Rayleigh channel whose elements are independent and identically distributed (iid) circularly symmetric zero mean complex Gaussian variables with unit variance denoted $C \mathcal{N}(0,1)$. Finally, the matrices $\boldsymbol{\Psi}_{r}=\boldsymbol{\Sigma}_{r} \otimes \boldsymbol{\Pi}_{r}$ and $\boldsymbol{\Psi}_{t}=\boldsymbol{\Sigma}_{t} \otimes \boldsymbol{\Pi}_{t}$ are the $2 N_{r} \times 2 N_{r}$ and $2 N_{t} \times 2 N_{t}$ composite transmit and receive correlation matrices, combing spatial and polarization correlations.

\section{B. Sub-Terahertz MIMO channel}

The channel at sub-THz bands suffers from high atmospheric attenuation and very strong obstruction losses (from walls, vegetation, furniture, etc.), and high sensitivity to the

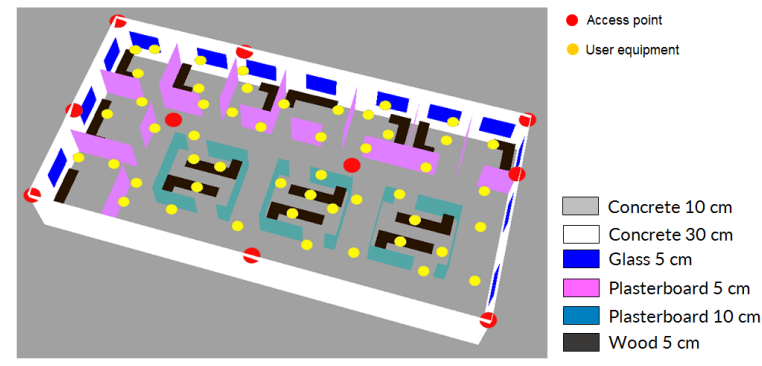

(a)

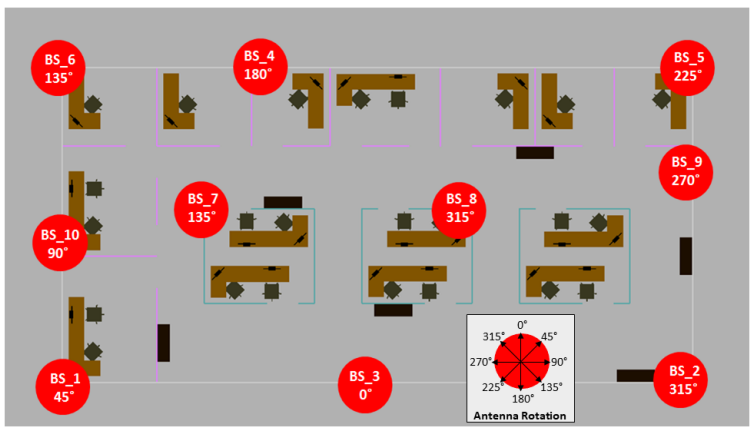

(b)

Fig. 2. In-door MIMO channel measurements [24]. (a) 3D physical positions of $10 \mathrm{AP}$ marked in red circles and 50 UEs marked in yellow. The office is composed of external walls, windows, internal walls, cubicle partitions and desks. (b) 2D positions of the $10 \mathrm{APs}$ and office furniture.

environment details (small objects, surface roughness, etc.). For clarification, most surfaces that appear smooth for microwave frequencies leads to significant diffuse scattering and strong specular reflections for sub-THz frequencies [23]. In this paper, we focus on short-range scenario such as kiosk or hot-spot where the atmospheric attenuation is irrelevant.

A ray-based deterministic channel modeling for sub- $\mathrm{THz}$ Band (mainly between $90-200 \mathrm{GHz}$ ) is presented in [24]. It is worth mentioning that the propagation channel model in [24] considers the material properties, gas attenuation and the impact of furniture that leads to more obstructions along the propagation paths and new scattered paths. In addition, it characterizes the main channel properties such as path loss and delay spread for LOS, NLOS with vegetation and NLOS cases for indoor in-office and outdoor in-street scenarios. In the following, we will focus on the downlink hotspot (or kiosk) indoor scenario where the Access Points (APs), acting as transmitters, and the user equipment (UE), acting as receivers without mobility, are equipped with $N_{t}$ and $N_{r}$ DP isotropic antennas respectively in the positions as depicted in Fig. 2. Indeed, Fig. 2 provides a description of a scenario where the 10 APs are marked in red and distributed throughout a typical single-floor office of size $20 \mathrm{~m} \times 10 \mathrm{~m}$, and the 50 UEs are highlighted with yellow circles. The office is composed of external walls, windows, internal walls, cubicle partitions ( 2 meters high), and desks. The APs are installed at realistic locations, i.e., on the wall or below the ceiling at $2.5 \mathrm{~m}$ height. Different realistic and random UE positions are considered in the different areas inside the office. Therefore, the MIMO propagation channels are obtained using the simulator for raybased deterministic channel modeling. 


\section{Sub-Terahertz RF impairments and challenges}

In this section, the main sub- $\mathrm{THz}$ band impairments and challenges are summarized. For high frequency broadband systems, the non-linearity of analog components used in Radio-Frequency (RF) front ends gives increased challenges in the modeling of circuits and in anticipating the compensation measures required for performance improvements. The RF front end is composed of all components between the antenna and the digital baseband system of a transceiver, namely mixer or modulator, phase shifter, and Power Amplifier (PA). Sub$\mathrm{THz}$ bands suffer from many RF challenges, especially for low cost implementation, and they can be summarized as follows:

- Efficiency and achievable transmit output power are low compared to sub-GHz

- Medium to high Phase noise of the LO that leads to significant degradation in Signal-to-Noise Ratio (SNR) and limits both Bit-Error Rate (BER) performance and throughput rate.

- High power consumption and limited resolution for ultrahigh sampling rate for ADCs [26]. bands, where the maximum achievable output power is in the order of 10 $\mathrm{dBm}$.

- Non-linearity effect of the PA and electronics components.

More precisely, the practical oscillator can never generate a pure sinusoid and the PN is more important at higher carrier frequencies. Thus, this impairment cannot be neglected since its impact is no more tolerable as in sub-GHz systems.

In general, the analysis of PN effect on MIMO systems is dependent on the RF architecture where there are mainly two LO setups: Distributed Oscillators (DO), Centralized or Common Oscillator (CO). The main difference is that each antenna in the DO setup has its own LO while in the CO setup all antennas at the transmitter|receiver are connected to a common LO. Consequently, all parallel streams in $\mathrm{CO}$ has the same PN while the DO suffers from different phase and amplitude distortions.

The PN model is widely investigated where the PN in general is modeled by two main models: Gaussian PN (uncorrelated PN) and Wiener PN (correlated PN). The Wiener model is more accurate since it includes the cumulative PN (correlated term) due to the integration step of the Phase Locked Loop (PLL). However, Wiener PN process becomes negligible compared to the Gaussian for wide-band systems where the oscillator corner frequency $f_{c}$ is small compared to the system bandwidth [5]. Thus uncorrelated Gaussian PN model is more appropriate to analyze sub-THz systems. Note that the uncorrelated model remains valid when the following condition is satisfied [5]:

$$
N_{s} f_{c}^{2} T^{2} \leq \frac{\ln (2)}{2 \pi^{2}}
$$

where $N_{s}$ is the number of symbols per frame, $f_{c}$ is the corner frequency of the oscillator and $T$ is the symbol period. It is clear that a careful communication system design can limit the Wiener effect by selecting the appropriate $N_{s}$ and $T$.
The received baseband vector of an equivalent $2 N_{r} \times 2 N_{t}$ MIMO system with phase noise can be expressed as:

$$
\tilde{\mathbf{y}}=\Phi_{r} \mathbf{H} \Phi_{t} \mathbf{x}+\mathbf{n},
$$

where $\Phi_{t}$ and $\Phi_{r}$ are the $2 N_{t} \times 2 N_{t}$ and $2 N_{r} \times 2 N_{r}$ diagonal matrices of phase noise from the transmitter and receiver oscillators respectively. These phase noise matrices can be represented as follows:

$$
\begin{aligned}
& \Phi_{t}=\operatorname{diag}\left(\left[e^{j \theta_{1}^{T x}}, \ldots, e^{j \theta_{2 N_{t}}^{T x}}\right]^{T}\right) \\
& \Phi_{r}=\operatorname{diag}\left(\left[e^{j \theta_{1}^{R x}}, \ldots, e^{j \theta_{2 N_{r}}^{R x}}\right]^{T}\right),
\end{aligned}
$$

where $\theta_{\iota}^{T x}$ and $\theta_{\kappa}^{R x}$ represent the PNs at the TAs and RAs respectively, and $\iota=\left\{1,2, \ldots, 2 N_{t}\right\}$ and $\kappa=\left\{1,2, \ldots 2 N_{r}\right\}$. The evenlodd values of $\iota$ and $\kappa$ are for the verticallhorizontal polarization, at the $\left\lfloor\frac{\ell-1}{2}\right\rfloor \mathrm{TA}$ and $\left\lfloor\frac{\kappa-1}{2}\right\rfloor$ RA respectively. These $\theta$ can be described in sub-THz band by a truncated Gaussian distribution $\mathcal{N}\left(0, \sigma_{g}^{2}\right)$ with zero-mean and variance $\sigma_{g}^{2}$ similar to SISO PN model described in [5]. In the DO setup, the PN at the different TA are independent, similarly at the receiver side. However, in the CO setup with independent oscillators for Tx and $\mathrm{Rx}$ sides (same oscillator is used for all antennas on each side but different oscillators for the Tx and $\mathrm{Rx}), \theta_{\iota}^{T x}=\theta^{T x}$ for all $\iota=\left\{1, \ldots, 2 N_{t}\right\}$ and $\theta_{\kappa}^{R x}=\theta^{R x}$ for all $\kappa=\left\{1, \ldots, 2 N_{r}\right\}$. Thus, the received signal in the $\mathrm{CO}$ setup can be simplified to:

$$
\tilde{\mathbf{y}}=e^{j \theta^{T x}} e^{j \theta^{R x}} \mathbf{H x}+\mathbf{n}
$$

\section{PERFORMANCE ANALYSIS}

In this section, we provide the theoretical Average Bit Error Probability (ABEP) analysis and we derive the upper bound of the ABEP which depends on the pairwise error probability (PEP). In addition, we provide validation through channel simulations.

\section{A. Average error probability bound}

The upper bound of the Average Bit Error Probability (ABEP), denoted by $P_{e}$, of the ML detection is obtained first by evaluating the average pairwise error probability (APEP), denoted by $\bar{P}_{s}$ and then averaging the set of ABEP for all possible pairs of symbols [29]. In fact, the pairwise error probability (PEP) is a function of fading channel coefficients and required to be averaged over the probability density function (pdf) of channel coefficients to provide the APEP, and finally to find the ABEP.

According to the union bounding technique, the ABEP of DP-GSM can be upper bounded by using the upper bounding relation [30]

$$
P_{e} \leq \frac{1}{2^{L}} \sum_{i=1}^{2^{L_{1}}} \sum_{\hat{i}=1}^{2^{L_{1}}} \sum_{\ell=1}^{2^{L_{2}}} \sum_{\hat{\ell}=1}^{2^{L_{2}}} \sum_{\nu=1}^{2^{L_{3}}} \sum_{\hat{v}=1}^{2^{L_{3}}} \frac{N(i, \hat{i}, \ell, \hat{\ell}, v, \hat{v})}{L} \bar{P}_{s}(i, \hat{i}, \ell, \hat{\ell}, v, \hat{v})
$$

where $N(i, \hat{i}, \ell, \hat{\ell}, v, \hat{v})$ is the number of bits in error between the respective channel/polarization and vector symbols pair $\left(\mathbf{H}_{I_{i}, P_{\ell}}, \mathbf{s}_{v}\right)$ and $\left(\mathbf{H}_{I_{\hat{i}}}, P_{\hat{\ell}}, \mathbf{s}_{\hat{v}}\right), \bar{P}_{s}(i, \hat{i}, \ell, \hat{\ell}, v, \hat{v})$ is the APEP, $L$ is the total number of bits (antenna, polarization and symbols), $2^{L}=N_{1} N_{2} M^{N_{a}}$ is the total number of configurations, 
and $N(i, \hat{i}, \ell, \hat{\ell}, v, \hat{v}) / L$ is summation weight for the pairwise probability.

To evaluate the conditional PEP analytically, we assume that an DP-GSM symbol consisting of the $i$-th TAC, $\ell$-th TPC, and the digitally modulated symbol vector $\mathbf{s}_{v}$ are transmitted. Due to noise and fading, it is detected in error as the TAC, TPC and digitally modulated symbol. Accordingly, the PEP representing the probability of incorrectly detecting $\mathbf{S}_{v}$ as $\mathbf{S}_{\hat{v}}$ conditioned on $\mathbf{H}$ is given by

$$
\begin{aligned}
& P_{S}(i, \hat{i}, \ell, \hat{\ell}, v, \hat{v})=\operatorname{Pr}(\mathbf{x} \rightarrow \hat{\mathbf{x}} \mid \mathbf{H}) \\
& =\operatorname{Pr}\left(\left\|\mathbf{y}-\mathbf{H}_{I, P} \mathbf{s}_{v}\right\|^{2}>\left\|\mathbf{y}-\mathbf{H}_{\hat{I}, \hat{P}} \mathbf{s}_{\hat{v}}\right\|^{2} \mid \mathbf{H}\right) \\
& =\operatorname{Pr}\left(\operatorname{tr}\left[-2 \mathcal{R}\left\{\left(\mathbf{H}_{I, P} \mathbf{s}_{v}-\mathbf{H}_{\hat{I}, \hat{P}} \mathbf{s}_{\hat{v}}\right) \mathbf{n}^{H}\right\}\right]\right. \\
& \left.\quad>\left\|\mathbf{H}_{I, P} \mathbf{s}_{v}-\mathbf{H}_{\hat{I}, \hat{P}} \mathbf{S}_{\hat{v}}\right\|_{F}^{2} \mid \mathbf{H}\right) \\
& =\operatorname{Pr}\left(-2 \mathcal{R}\left\{\mathbf{n}^{H}\left(\mathbf{H}_{I, P} \mathbf{S}_{v}-\mathbf{H}_{\hat{I}, \hat{P}} \mathbf{S}_{\hat{v}}\right)\right\}\right. \\
& \left.\quad>\left\|\mathbf{H}_{I, P} \mathbf{s}_{v}-\mathbf{H}_{\hat{I}, \hat{P}} \mathbf{S}_{\hat{v}}\right\|_{F}^{2} \mid \mathbf{H}\right)
\end{aligned}
$$

where $2 \mathcal{R}\left\{\mathbf{n}^{H}\left(\mathbf{H}_{I, P} \mathbf{S}_{v}-\mathbf{H}_{\hat{I}, \hat{P}} \mathbf{S}_{\hat{v}}\right)\right\}$ is a real Gaussian random variable with zero mean and variance $2 \sigma^{2}\left\|\mathbf{H}_{I, P} \mathbf{S}_{v}-\mathbf{H}_{\hat{I}, \hat{P}} \mathbf{S}_{\hat{v}}\right\|_{F}^{2}$ (see Appendix A for more details). Therefore, the probability that $\mathbf{x}$ is transmitted but $\mathbf{x} \neq \hat{\mathbf{x}}$ is given by

$$
\operatorname{Pr}(\mathbf{x} \rightarrow \hat{\mathbf{x}} \mid \mathbf{H})=Q\left(\sqrt{\frac{\left\|\mathbf{H}_{I, P} \mathbf{S}_{v}-\mathbf{H}_{\hat{I}, \hat{P}} \mathbf{S}_{\hat{v}}\right\|_{F}^{2}}{2 \sigma^{2}}}\right)
$$

where $Q($.$) is the Gaussian Q-function.$

The average PEP is given by

$$
\operatorname{Pr}(\mathbf{x} \rightarrow \hat{\mathbf{x}})=\mathbb{E}_{\mathbf{H}}\left\{Q\left(\sqrt{\frac{\left\|\mathbf{H}_{I, P} \mathbf{S}_{v}-\mathbf{H}_{\hat{I}, \hat{P}} \mathbf{S}_{\hat{v}}\right\|_{F}^{2}}{2 \sigma^{2}}}\right)\right\}
$$

Defining a new vector $\mathbf{z}=\frac{1}{\sqrt{2 \sigma^{2}}}\left(\mathbf{H}_{I, P} \mathbf{S}_{v}-\mathbf{H}_{\hat{I}, \hat{P}} \mathbf{S}_{\hat{v}}\right) \quad \in$ $\mathbb{C}^{2 N_{r} \times 1}$, the previous equation is equivalent to

$$
\begin{aligned}
\operatorname{Pr}(\mathbf{x} \rightarrow \hat{\mathbf{x}}) & =\mathbb{E}_{\mathbf{z}}\left\{Q\left(\sqrt{\|\mathbf{z}\|_{F}^{2}}\right)\right\} \\
& =\frac{1}{\pi} \int_{0}^{\pi / 2} \int_{\mathbf{z}} \exp \left(-\frac{\mathbf{z}^{H} \mathbf{z}}{2 \sin ^{2} \theta}\right) f_{\mathbf{z}}(\mathbf{z}) \mathrm{d} \mathbf{z} \mathrm{d} \theta
\end{aligned}
$$

where $f_{\mathbf{z}}(\mathbf{z})$ is the pdf of $\mathbf{z}$ which is a proper complex Gaussian vector as defined in [27] with pdf given as

$$
f_{\mathbf{z}}(\mathbf{z})=\frac{1}{\pi^{2 N r}\left|\mathbf{\Sigma}_{\mathbf{z}}\right|} \exp \left(-\left(\mathbf{z}-\mathbf{m}_{\mathbf{z}}\right)^{H} \mathbf{\Sigma}_{\mathbf{z}}^{-1}\left(\mathbf{z}-\mathbf{m}_{\mathbf{z}}\right)\right) .
$$

According to the channel model $\mathbf{H}$ given by (17), the expression of $\mathbf{z}$ is given by

$$
\begin{aligned}
& \mathbf{z}=\sqrt{\frac{K}{2 \sigma^{2}(1+K)}}\left(\left(\mathbf{1}_{N_{r} \times N_{t}} \otimes \boldsymbol{\Gamma}\right) \odot \mathbf{H}_{\mathrm{LOS}}\right)(\mathbf{x}-\hat{\mathbf{x}}) \\
& +\sqrt{\frac{1}{2 \sigma^{2}(1+K)}}\left(\left(\mathbf{1}_{N_{r} \times N_{t}} \otimes \boldsymbol{\Gamma}\right) \odot\left(\boldsymbol{\Psi}_{r}^{\frac{1}{2}} \mathbf{H}_{\mathrm{NLOS}} \boldsymbol{\Psi}_{t}^{\frac{1}{2}}\right)\right)(\mathbf{x}-\hat{\mathbf{x}}) .
\end{aligned}
$$

As a consequence, the expectation $\mathbf{m}_{\mathbf{z}}=\mathbb{E}\{\mathbf{z}\}$ and the covariance matrix $\boldsymbol{\Sigma}_{\mathbf{z}}=\mathbb{E}\left\{\left(\mathbf{z}-\mathbf{m}_{\mathbf{z}}\right)\left(\mathbf{z}-\mathbf{m}_{\mathbf{z}}\right)^{H}\right\}$ are given by

$$
\begin{aligned}
& \mathbf{m}_{\mathbf{z}}=\sqrt{\frac{K}{2 \sigma^{2}(1+K)}}\left(\left(\mathbf{1}_{N_{r} \times N_{t}} \otimes \boldsymbol{\Gamma}\right) \odot \mathbf{H}_{\mathrm{LOS}}\right)(\mathbf{x}-\hat{\mathbf{x}}) \\
& =\sqrt{\frac{K}{2 \sigma^{2}(1+K)}\left((\mathbf{x}-\hat{\mathbf{x}})^{T} \otimes \mathbf{I}_{2 N r}\right) \operatorname{vec}\left(\mathbf{1}_{N_{r} \times N_{t}} \otimes \boldsymbol{\Gamma}\right) \odot} \\
& \operatorname{vec}\left(\mathbf{H}_{\mathrm{LOS}}\right)
\end{aligned}
$$

$$
\begin{aligned}
& \boldsymbol{\Sigma}_{\mathbf{z}}=\frac{1}{2 \sigma^{2}(1+K)}\left((\mathbf{x}-\hat{\mathbf{x}})^{T} \otimes \mathbf{I}_{2 N r}\right) \operatorname{vec}\left(\mathbf{1}_{N_{r} \times N_{t}} \otimes \boldsymbol{\Gamma}\right) \odot \\
& \left(\boldsymbol{\Psi}_{t}^{T} \otimes \boldsymbol{\Psi}_{r}\right) \odot \operatorname{vec}\left(\mathbf{1}_{N_{r} \times N_{t}} \otimes \boldsymbol{\Gamma}\right)^{H}\left((\mathbf{x}-\hat{\mathbf{x}})^{T} \otimes \mathbf{I}_{2 N r}\right)^{H} .
\end{aligned}
$$

Finally, using the distribution of $\mathbf{z}$ and following the methodology of [28] to compute the double integral in equation (32), the APEP $\operatorname{Pr}(\mathbf{x} \rightarrow \hat{\mathbf{x}})$ is then given by (see Appendix B for details)

$$
\operatorname{Pr}(\mathbf{x} \rightarrow \hat{\mathbf{x}})=\frac{1}{\pi} \int_{0}^{\pi / 2} \frac{\exp \left(-\mathbf{m}_{\mathbf{z}}^{H}\left(\boldsymbol{\Sigma}_{\mathbf{z}}+2 \sin ^{2} \theta \mathbf{I}\right)^{-1} \mathbf{m}_{\mathbf{z}}\right)}{\left|\frac{\boldsymbol{\Sigma}_{\mathbf{z}}}{2 \sin ^{2} \theta}+\mathbf{I}\right|} \mathrm{d} \theta
$$

The closed-form expression of the integral form of the APEP is not available. In this case, the APEP is upper bounded by assuming

$$
\operatorname{Pr}(\mathbf{x} \rightarrow \hat{\mathbf{x}}) \leq \frac{\exp \left(-\mathbf{m}_{\mathbf{z}}^{H}\left(\boldsymbol{\Sigma}_{\mathbf{z}}+2 \mathbf{I}\right)^{-1} \mathbf{m}_{\mathbf{z}}\right)}{\left|\frac{\mathbf{\Sigma}_{\mathbf{z}}}{2}+\mathbf{I}\right|}
$$

\section{B. Simulation results}

In this subsection, we provide the performance of the DP-GSM evaluated through Monte Carlo simulations of the average bit-error-rate (BER) and different values of signalto-noise ratio (SNR) and compared to the theoretical ABEP upper bounds. These Monte Carlo simulations consider the average BER over 200 channel realizations, where $10^{5}$ DPGSM symbols are evaluated for each channel realization. The simulations are used over correlated Rayleigh and Rician channel with different values of $\beta$ according to the mathematical model given by (17) and also over sub-THz channels shown in subsection III-B. Different comparisons between our proposed DP-GSM system and the DP-SMX system for several parameters and different channel conditions are also provided and discussed.

The proposed DP-GSM system is evaluated using the amplitude and phase modulation (APM): QPSK. The latter has shown a good performance with UP-GSM system comparing to the other modulations such as the DPSK and the 4-PAM [21] and compared to UP-SMX system in sub-THz environment [6]. For our case of study, we choose the configuration $\left(N_{t}=5, N_{a}=3, N_{r}=\{3,5\}\right)$. The corresponding $\mathrm{SE}$ is in the order of $12 \mathrm{bpcu}$. Moreover, the corresponding data rate is estimated with a total system bandwidths of $50 \mathrm{GHz}$ which is available in the band between $90 \mathrm{GHz}$ and $200 \mathrm{GHz}$. The current configuration for DP-GSM systems can reach up to 500 Gbps (0.5 Tbps) when the considered total system bandwidth, after channel aggregation and bounding is $50 \mathrm{GHz}$ with 0.2 rolloff factor for the pulse shaping. The simulation parameters 
TABLE II

SIMULATION PARAMETERS AND A SUB-THZ SYSTEM EXAMPLE.

\begin{tabular}{|c|c|}
\hline APM & DP-GSM configuration \\
\hline QPSK & $N_{t}=5, N_{a}=3, N_{r}=\{3,5\}$ \\
\hline Parameters for sub-THz & Value \\
System example & 150 \\
\hline Carrier frequency (GHz) & 0.50 \\
Channel bandwidth (GHz) & 12 \\
Spectral Efficiency (bpcu) & Root Raise cosine:0.2 \\
Pulse Shaping: Rolloff & 10 \\
Spectral efficiency (b/s/Hz) & 5 \\
Data Rates per Channel (Gbps) & 500 \\
Data Rates with 50 GHz (Gbps) & $\sigma_{g}^{2}$ Value \\
\hline RF-impairments: PN & 0.001 \\
\hline Low & 0.01 \\
Medium & 0.1 \\
Strong &
\end{tabular}

and the data rates estimation using DP-GSM are summarized in table II. In all our simulations, the Rician factor $K$ is set to 3 and the polarization correlation for the transmit and the receive components are chosen identical $\left|\gamma_{t}\right|=\left|\gamma_{r}\right|=0.1$.

First, to further illustrate the potential of DP-GSM with the MOB-MMSE detector, the ML detector is compared with our approach. Fig. 3, shows the simulation results of the DP-GSM system over correlated Rayleigh channel (a) and correlated Rician channel (b) for QPSK with MOB-MMSE and ML detectors. The used spatial correlation values are $\beta=\{0.2,0.8\}$ corresponding, respectively, to a low and a strong spatial correlation, while a $\mu=0.2$ leakage parameter is selected corresponding to a realistic XPD value equal to 4 . However, a full study of the leakage parameter $\mu$ is given in Bouhlel et al. [22] where other values of $\mu$ have been experimented. As it is shown in Fig. 3 there is quite acceptable difference of $(1-2.5) \mathrm{dB}$ between ML and MOB-MMSE at the BER of $10^{-4}$. Thus, the MOB-MMSE for DP-GSM system can achieve sub-optimal performance with low complexity. The same behavior and performance difference between these two detectors is also observed with DP-GSM using higher-order modulation in Fig. 4, where the performance of the DP-GSM using 16QAM is evaluated over correlated Rayleigh channel (see Fig. 4.(a)) and correlated Rician channel (see Fig. 4.(b)). Note that the DP-GSM configuration in Fig. 4 with 16QAM is chosen as follows: $N_{t}=4, N_{a}=2$ and $N_{r}=3$, that leads to $\mathrm{SE}=12 \mathrm{bpcu}$.

In the second experiment, the DP-SMX and the UP-GSM are compared with the DP-GSM system. We recall the reader that the SMX is a particular case of the GSM technique where all the TAs are activated to transmit simultaneously the APM symbols. In the DP-SMX system, all TAs and RAs use the dual polarization to convey simultaneously different symbols on each polarization. The comparison is made using different spatial correlation configurations, $\beta \in\{0.2,0.8\}$, and under different fading channels, Rayleigh and Rician channel. The comparison is made using the optimal ML detector where the number of antennas for the compared systems: DP-GSM, DP-SMX, and UP-GSM are respectively: $\left(N_{t}=5, N_{a}=3, N_{r}=3\right),\left(N_{t}=3, N_{r}=3\right)$, and $\left(N_{t}^{U P}=10, N_{a}=3, N_{r}^{U P}=6\right)$ to have the same SE given by $12 \mathrm{bpcu}$ for a fair comparison. It is worth mentioning that with

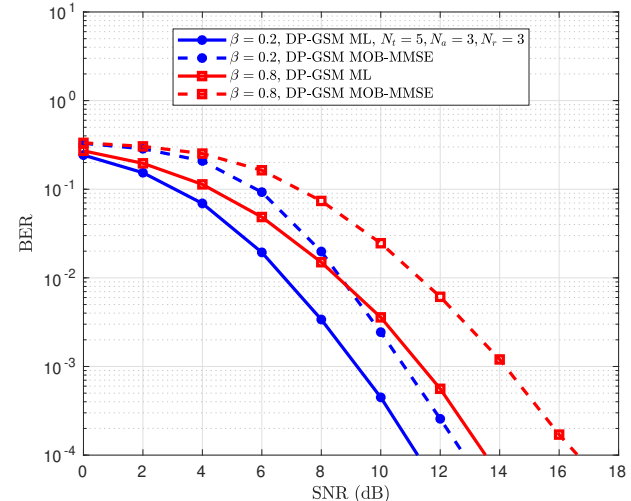

(a)

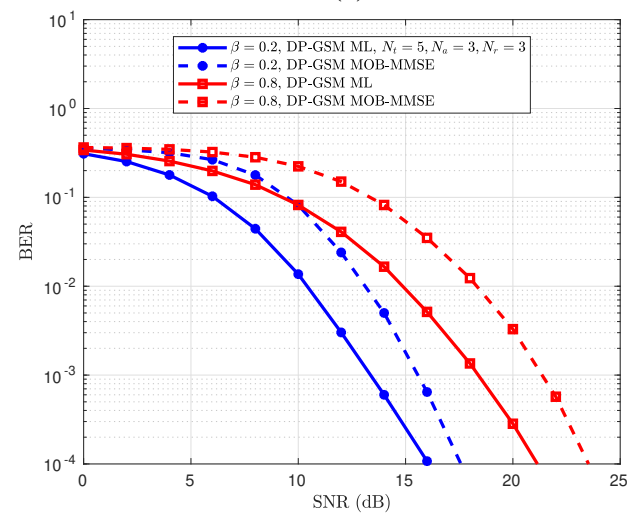

(b)

Fig. 3. BER performance of $N_{r} \times N_{t}$ DP-GSM system with spatial correlation $\beta \in\{0.2,0.8\}$ and power leakage parameter $\mu=0.2$ for QPSK over: (a) correlated Rayleigh channel and (b) correlated Rician channel $(K=3)$ using both MOB-MMSE and ML detectors. The SE is $12 \mathrm{bpcu}$.

these configurations DP-SMX activates the double number of TA polarizations compared to DP-GSM to achieve the same SE, because DP-SMX uses both polarization to transmit different symbols simultaneously while DP-GSM uses only one polarization with each activated TA. It can be seen in Fig. 5 that the DP-GSM outperforms the UP-GSM system in both channels and both spatial correlations. In all cases, the DP-GSM allows to have a more compact antenna array physical size compared to UP-GSM, which is crucial for UE. Figure 5 shows clearly that the DP-GSM and the DP-SMX have the same performance with the ML detector in low spatial correlation and for both fading channels. In the context of high spatial correlation, the DP-GSM outperforms the DP-SMX in both channels and the SNR difference between them is about $1-1.5 \mathrm{~dB}$ at a $\mathrm{BER}=10^{-4}$. This can be explained by the fact that the inter-antenna interference in the DP-SMX system is more important than in the DP-GSM since the first system transmits $2 N_{t}=6$ different APM symbols simultaneously by using both polarization. However, the DP-GSM system emits $N_{a}=3$ different APM symbols by using only one polarization with each activated TA. This lower inter-antenna interference of DP-GSM helps to achieve better performance at high spatial correlation. This result implies that employing DP antennas enhances the robustness of the GSM system even further and leads to better performance than its DP-SMX counterpart at high spatial correlation. In addition, this result reveals that the virtual bits conveyed via the indexation of TAPC in DP-GSM 


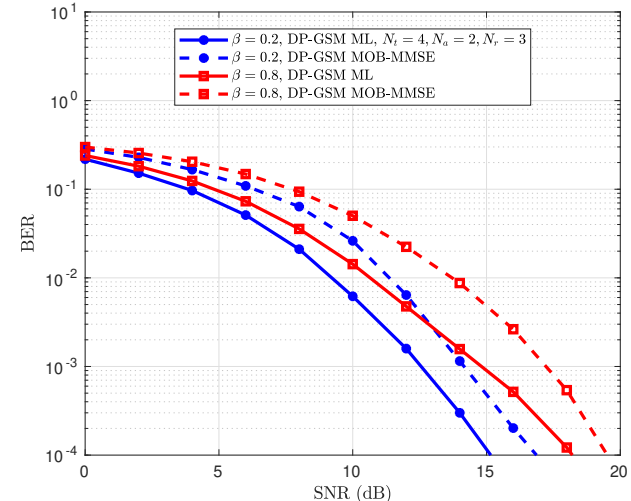

(a)

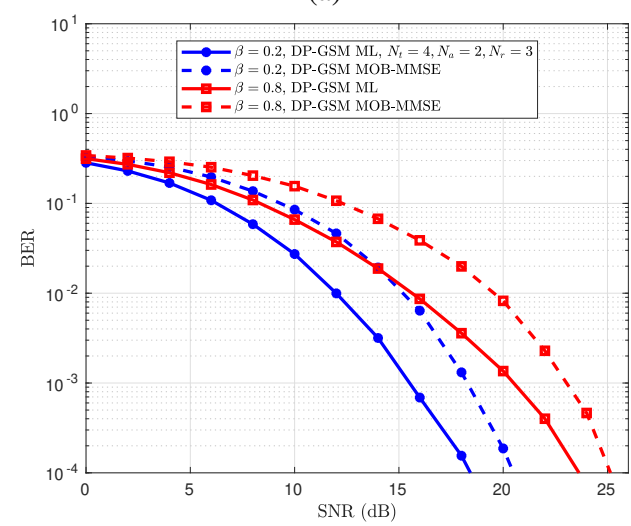

(b)

Fig. 4. BER performance of $N_{r} \times N_{t}$ DP-GSM system with spatial correlation $\beta \in\{0.2,0.8\}$ and power leakage parameter $\mu=0.2$ for 16QAM over: (a) correlated Rayleigh channel and (b) correlated Rician channel $(K=3)$ using both MOB-MMSE and ML detectors. The SE is $12 \mathrm{bpcu}$.

are more robust than transmitting only APM symbols even at high spatial correlation.

Fig. 5 shows also that the derived ABEP upper bounds accurately match with the simulation results of the DP-GSM especially in the high SNR range.

The third experiment consists to study the performance of the DP-GSM system using the sub-THz phase noise model described in the previous section. The study is also extended to the case of DP-SMX for fair comparison with our system and performed with different PN levels (low $\sigma_{g}^{2}=0.001$, medium $\sigma_{g}^{2}=0.01$, strong $\sigma_{g}^{2}=0.1$ ) without applying any phase noise mitigation technique. Figure 6 and 7 show these comparisons between DP-GSM and DP-SMX systems for QPSK over correlated Rayleigh channel and correlated Rician channel $(K=3)$ respectively with spatial correlation (a) $\beta=0.2$ and (b) $\beta=0.8$. It is clear that the performance for both system are very close especially when the fading channel is Rayleigh and the spatial correlation is small $(\beta=0.2)$. When this value increases, the DP-GSM outperforms the DPSMX by 1.5 to $2.5 \mathrm{~dB}$ with low and medium PN respectively. It is also worth to mention that the performance for both systems degrade considerably for strong phase noise. In the case of Rician fading channel (Figure 7), the performance of both systems is acceptable for low value of spatial correlation but when it increases, the performance for both systems is degraded especially for medium and strong phase noise.

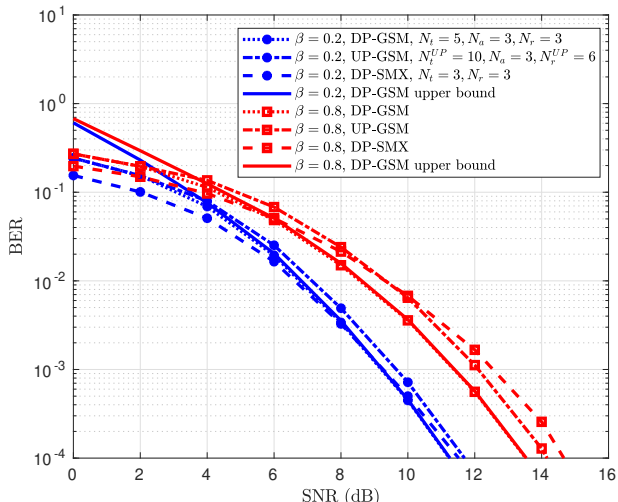

(a)

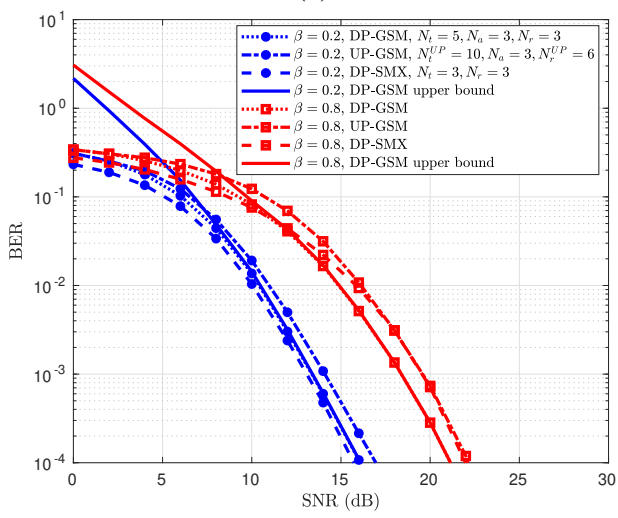

(b)

Fig. 5. BER performance comparison of DP-GSM, DP-SMX, and UP-GSM systems with spatial correlation $\beta \in\{0.2,0.8\}$ and power leakage $\mu=0.2$ over: (a) correlated Rayleigh channel and (b) correlated Rician channel $(K=$ 3) for QPSK using ML detector. The SE is $12 \mathrm{bpcu}$.

\section{Results with real sub-THz MIMO channel}

In this subsection we extended our analysis using a more realistic sub-THz channel different from that presented in (17). In hereafter we compare the performance of the DP-SMX and the UP-GSM systems using a ray-based deterministic channel modelling for sub- $\mathrm{THz}$ band $(90-200 \mathrm{GHz})$ developed in [24]. We adopt here the downlink hotspot (or kiosk) indoor scenario generated using the ray-based channel model described in Section III-B. Here, Uniform-Linear-Array (ULA) is considered with antenna element separation of $4 \lambda$ for a lower spatial correlation [25], where $\lambda$ is the wavelength. Also, different channel realizations are considered by using the 10 APs marked in red and distributed throughout the room, and the 50 UEs highlighted with yellow circles in Fig. 2. The considered range for UE is going from $2 \mathrm{~m}$ to $8 \mathrm{~m}$, i.e., we use in average a $5 \mathrm{~m}$ separation distance between UE and AP.

For a fair comparison, the DP-SMX and the UP-GSM are compared with the DP-GSM system under the same SE which is $12 \mathrm{bpcu}$ using same detector, same number of RA, same APM for all systems, and same number of activated TA for UP-GSM and DP-GSM. The following configurations are adopted: for DP-GSM $\left(N_{t}=5, N_{a}=3, N_{r}=3\right)$, for DP$\operatorname{SMX}\left(N_{t}=3, N_{r}=3\right)$ and UP-GSM $\left(N_{t}^{U P}=10, N_{a}=3\right.$, $\left.N_{r}^{U P}=6\right)$. Figure 8 , shows such a comparison using the ML detector for the three systems. It can be seen that the DPGSM and DP-SMX have nearly the same performance and 


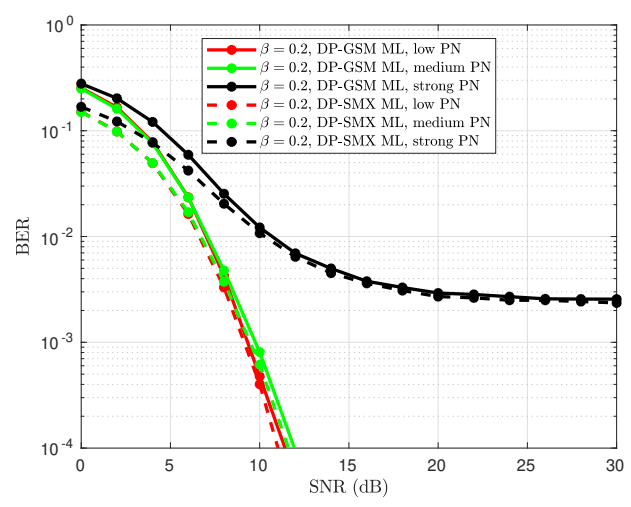

(a)

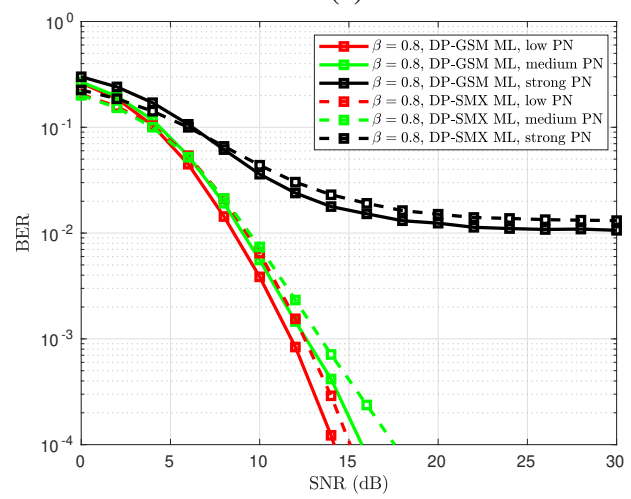

(b)

Fig. 6. BER performance of DP-GSM and DP-SMX systems for QPSK over correlated Rayleigh channel with spatial correlation: (a) $\beta=0.2$ and (b) $\beta=0.8$ using ML detector. Both systems are affected by phase noise with different levels (low, medium and strong noise). The power leakage parameter is $\mu=0.2$ and the SE is $12 \mathrm{bpcu}$.

they outperform the UP-GSM with a difference of $1 \mathrm{~dB}$ at the BER of $10^{-4}$ (see solid lines). Moreover, when the number of DP RAs increases: $N_{r}=5$ for both DP-GSM and DPSMX, and $N_{r}^{U P}=10$ for UP-GSM, the performances of the three systems improve and the DP-GSM and DP-SMX systems always keep their superiority compared to UP-GSM.

Other effects related to sub- $\mathrm{THz}$ impairment can be observed in Fig. 9, where different phase noise levels are used and different number of received DP antennas $N_{r}=3$ in Fig. 9a, and $N_{r}=5$ in Fig. 9b are used for comparing the performances between the DP-GSM and the DP-SMX systems. Fig. 9, shows clearly that both systems achieve same performance for any value of $N_{r}$ with low PN. The gain achieved by the DP-SMX is slightly higher (about $1.5 \mathrm{~dB}$ at a BER of $10^{-4}$ ) with medium PN and $N_{r}=3$ when is compared with the DP-GSM. However, it is important to highlight that both systems fail to detect correctly the conveyed bits in their respective systems and suffer from a high error floor when they experiment a strong PN impairment even when the value of $N_{r}$ is high, because the system considers uncoherent detection without any PN cancellation. When the number of received DP antennas increases to $N_{r}=5$, the performance of the DPGSM and the DP-SMX systems improves compared to the case where $N_{r}=3$ and the performances of both systems become similar. By comparing Fig. 9a and Fig. 9b, we can notice that the DP-GSM system register a gain of $2 \mathrm{~dB}$ at

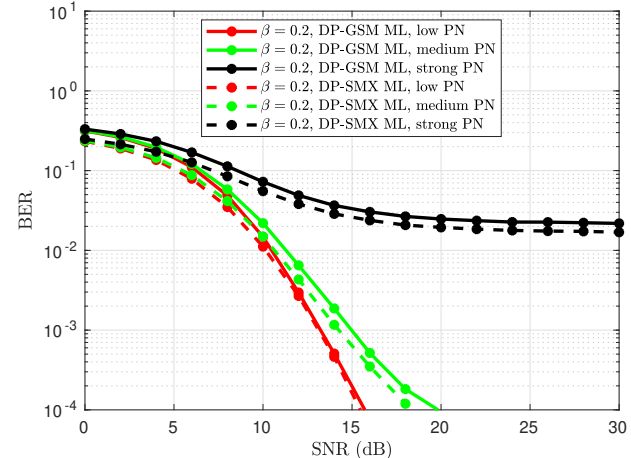

(a)

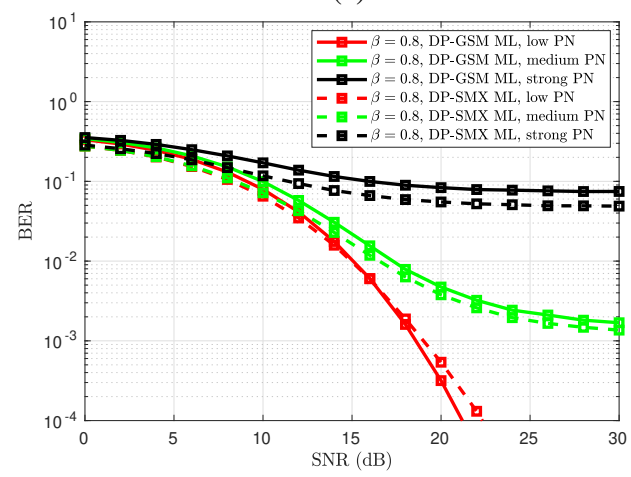

(b)

Fig. 7. BER performance of DP-GSM and DP-SMX systems for QPSK over correlated Rician channel $(K=3)$ with spatial correlation: (a) $\beta=0.2$ and (b) $\beta=0.8$ using ML detector. Both systems are affected by phase noise with different levels (low, medium and strong noise). The power leakage parameter is $\mu=0.2$ and the SE is $12 \mathrm{bpcu}$.

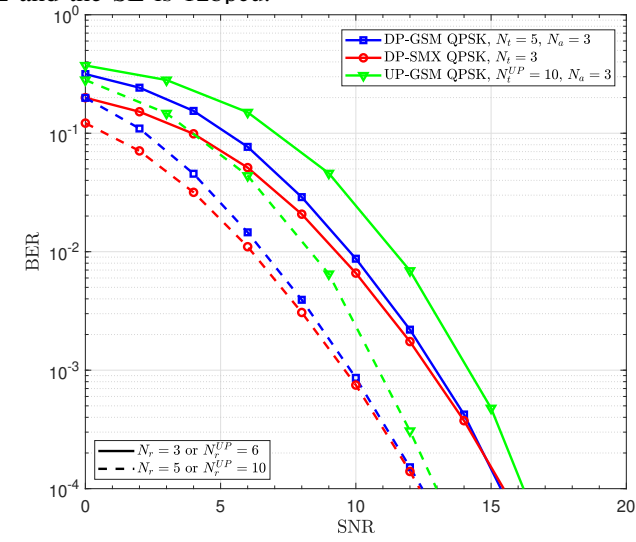

Fig. 8. BER performance of DP-GSM, DP-SMX and UP-GSM systems from sub- $\mathrm{THz}$ measurements for QPSK using ML detector. The concerned sub$\mathrm{THz}$ indoor MIMO channels are generated using ULA array geometry with $4 \lambda$ antenna separation. The SE is $12 \mathrm{bpcu}$. The AP-UE antenna separation is $d=[2 m, 8 m]$ with $d_{\text {mean }}=5 m$.

$\mathrm{BER}=10^{-4}$ at low $\mathrm{PN}$ and a gain of $3.5 \mathrm{~dB}$ at medium PN.

From indicated above, it can be concluded that the DPGSM system with QPSK is robust in low and medium PN impairments and can provide interesting performances in sub$\mathrm{THz}$ channel, however, all considered systems fail under strong $\mathrm{PN}$ value.

\section{CONCLUSION}

In this paper, the DP-GSM system is proposed and evaluated over correlated Rayleigh/Rician channels and sub-THz chan- 


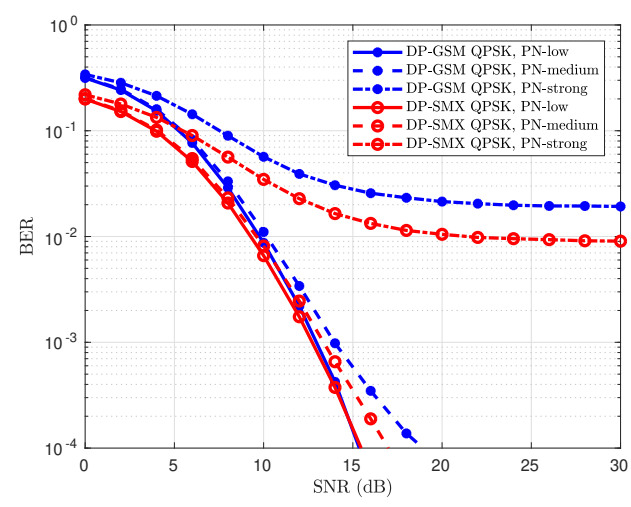

(a)

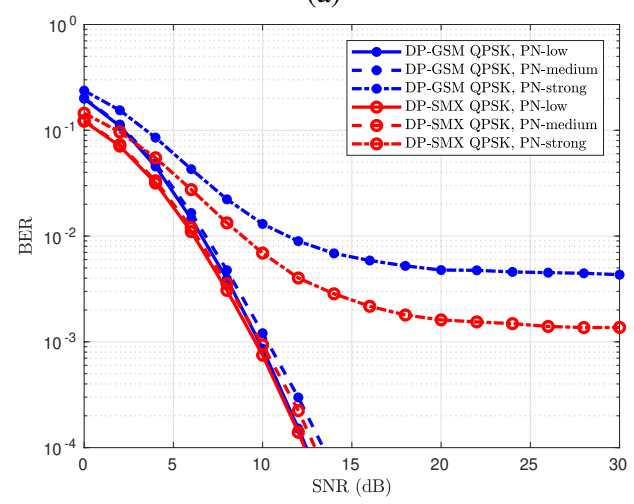

(b)

Fig. 9. BER performance of DP-GSM and DP-SMX systems with sub-THz impairments. The SE is $12 \mathrm{bpcu}$ and both systems are affected by phase noise with different levels (low, medium and strong). The sub-THz indoor MIMO channels are generated using ULA array geometry with $4 \lambda$ antenna separation. The AP-UE antenna separation is $d=[2 \mathrm{~m}, 8 \mathrm{~m}]$ with $d_{\text {mean }}=5 \mathrm{~m}$. (a) $N_{r}=3$ and (b) $N_{r}=5$

nels with/without RF-impairments such as the phase noise. The proposed system exploits the polarization IM dimension to increase the SE of conventional GSM system by $N_{a} \log _{2}\left(N_{p}\right)$. In addition, a low-complexity MOB-MMSE is proposed for DP-GSM to detect the transmitted complex symbols, the activated antennas' indices, and their polarizations. Theoretical analysis and Monte-Carlo simulations reveal that the DPGSM system provides a $1 \mathrm{~dB}$ gain compared to the UP-GSM system. It is also shown that the proposed DP-GSM scheme is more robust against the Rician fading effects with interantenna correlation, and therefore beneficial for improving the overall SE. Besides, the proposed scheme has demonstrated its good performance with RF-impairments even with the medium phase noise level. More precisely, the DP-GSM outperforms the DP-SMX in Rayleigh channel by 1.5 to $2.5 \mathrm{~dB}$ with low and medium PN, respectively. Moreover, the depicted results using the ray-based deterministic channel model for the subTHz band $(90-200 \mathrm{GHz})$ with RF-impairments reveal that the DP-GSM performance with low and medium PN levels are very close and they are similar to that of DP-SMX scheme. Note that DP-GSM, like any other transmit spatial modulation scheme, can use either RF-switching or full-RF-based transmitter architectures. The former has a lower transmitter cost ( $N_{a}$ RF chains instead of $2 N_{t}$ with $N_{t}$ DP antennas), but at the price of SE degradation [31], [32], while the latter architecture doesn't have any SE degradation or switching rate limitation, but it leads to the same transmitter cost like DP-SMX. However, DP-GSM receiver in all cases can have a lower cost since the receiver of DP-GSM can use only $N_{a} \leq N_{t}$ DP RAs ( $2 N_{a}$ RF chains) while DP-SMX receiver requires at least $N_{t}$ DP RAs ( $2 N_{t} \mathrm{RF}$ chains). It is worth mentioning that DP-GSM also has many advantages compared to DP-SMX, such as: (1) lower inter-antenna interference due to transmitting less number of APM symbols for the same SE; (2) higher robustness to high spatial correlation; (3) DP-GSM consumes less power compared to DP-SMX when the same transmit power per APM symbol is used with both systems because a lower number of APM symbols are needed with DPGSM compared to DP-SMX to achieve the same SE. These advantages are very important in sub-THz bands with high spatial correlation and limited transmit output power. This paper provides more advances in a path still full of challenges for real MIMO ultra-high data rate wireless systems in sub$\mathrm{THz}$ bands. For instance, the proposed system can be easily extended to use slanted linear polarization that conserves the orthogonality between both polarization planes, and the slanted polarization can help more to have better beam-alignment with UE for a maximum gain. Finally, it is worth mentioning that an appealing spectral and energy-efficient IM domain, called Filter IM domain, is explored recently by introducing Filter Shapes Index Modulation (FSIM) [40], and MIMO-FISM that had also shown significant advantages for sub-THz bands [41].

\section{APPENDIX A}

The conditional pdf of the received signal is given by

$$
\begin{aligned}
f_{\mathbf{y}}(\mathbf{y} \mid \mathbf{H}, \mathbf{x})=\prod_{i=1}^{2 N_{r}} f_{n_{i}}\left(n_{i}\right) & =\prod_{i=1}^{2 N_{r}} \frac{1}{\pi \sigma^{2}} \exp \left(-\frac{\left|n_{i}\right|^{2}}{\sigma^{2}}\right) \\
& =\frac{1}{\pi^{2 N_{r}} \sigma^{4 N_{r}}} \exp \left(-\frac{\|\mathbf{n}\|_{F}^{2}}{\sigma^{2}}\right) .
\end{aligned}
$$

The ML solution is given by

$$
\begin{aligned}
\hat{\mathbf{x}} & =\arg \max _{\mathbf{x}} f_{\mathbf{y}}(\mathbf{y} \mid \mathbf{H}, \mathbf{x}) \\
& =\arg \max _{\mathbf{x}} \frac{1}{\pi^{2 N_{r}} \sigma^{4 N_{r}}} \exp \left(-\frac{1}{\sigma^{2}} \operatorname{tr}\left[(\mathbf{y}-\mathbf{H x})(\mathbf{y}-\mathbf{H x})^{H}\right]\right) \\
& =\arg \min _{\mathbf{x}} \operatorname{tr}\left[(\mathbf{y}-\mathbf{H x})(\mathbf{y}-\mathbf{H x})^{H}\right] .
\end{aligned}
$$

Accordingly,

$$
\left(\hat{i}, \hat{\ell}, \mathbf{s}_{\hat{v}}\right)=\arg \min _{i, \ell, v} \operatorname{tr}\left[\left(\mathbf{y}-\mathbf{H}_{I, P} \mathbf{s}_{v}\right)\left(\mathbf{y}-\mathbf{H}_{I, P} \mathbf{S}_{v}\right)^{H}\right] .
$$

For erroneous detected vector symbol $\left(\hat{i}, \hat{\ell}, \mathbf{s}_{\hat{v}}\right) \neq\left(i, \ell, \mathbf{s}_{v}\right)$ the following condition is satisfied

$\operatorname{tr}\left[\left(\mathbf{y}-\mathbf{H}_{I, P} \mathbf{s}_{v}\right)\left(\mathbf{y}-\mathbf{H}_{I, P} \mathbf{s}_{v}\right)^{H}\right] \geq \operatorname{tr}\left[\left(\mathbf{y}-\mathbf{H}_{\hat{I}, \hat{P}} \hat{\mathbf{s}}_{\hat{v}}\right)\left(\mathbf{y}-\mathbf{H}_{\hat{I}, \hat{P}} \mathbf{s}_{\hat{v}}\right)^{H}\right]$

The previous inequality can be written otherwise

$$
\begin{aligned}
\operatorname{tr}\left[\mathbf{n} \mathbf{n}^{H}\right] & \geq \operatorname{tr}\left[\left(\mathbf{y}-\mathbf{H}_{\hat{I}, \hat{P}} \mathbf{s}_{\hat{v}}\right)\left(\mathbf{y}-\mathbf{H}_{\hat{I}, \hat{P}} \mathbf{s}_{\hat{v}}\right)^{H}\right] \\
& =\operatorname{tr}\left[\left(\left(\mathbf{H}_{I, P} \mathbf{s}_{v}-\mathbf{H}_{\hat{I}, \hat{P}} \mathbf{s}_{\hat{v}}\right)+\mathbf{n}\right)\left(\left(\mathbf{H}_{I, P} \mathbf{S}_{v}-\mathbf{H}_{\hat{I}, \hat{P}} \mathbf{s}_{\hat{v}}\right)+\mathbf{n}\right)^{H}\right] \\
& =\operatorname{tr}\left[\left(\mathbf{H}_{I, P} \mathbf{s}_{v}-\mathbf{H}_{\hat{I}, \hat{P}} \mathbf{s}_{\hat{v}}\right)\left(\mathbf{H}_{I, P} \mathbf{s}_{v}-\mathbf{H}_{\hat{I}, \hat{P}} \mathbf{s}_{\hat{v}}\right)^{H}\right]+ \\
& \operatorname{tr}\left[2 \mathcal{R}\left\{\left(\mathbf{H}_{I, P} \mathbf{s}_{v}-\mathbf{H}_{\hat{I}, \hat{P}} \mathbf{s}_{\hat{v}}\right) \mathbf{n}^{H}\right\}\right]+\operatorname{tr}\left[\mathbf{n} \mathbf{n}^{H}\right] .
\end{aligned}
$$


The last inequality implies :

$$
\begin{aligned}
\operatorname{tr}\left[-2 \mathcal{R}\left\{\left(\mathbf{H}_{I, P} \mathbf{s}_{v}-\mathbf{H}_{\hat{I}, \hat{P}} \mathbf{s}_{\hat{v}}\right) \mathbf{n}^{H}\right\}\right] & \geq \operatorname{tr}\left[\left(\mathbf{H}_{I, P} \mathbf{s}_{v}-\mathbf{H}_{\hat{I}, \hat{P}} \mathbf{s}_{\hat{v}}\right) \times\right. \\
& \left.\left(\mathbf{H}_{I, P} \mathbf{s}_{v}-\mathbf{H}_{\hat{I}, \hat{P}} \mathbf{S}_{\hat{v}}\right)^{H}\right] \\
& =\left\|\mathbf{H}_{I, P} \mathbf{s}_{v}-\mathbf{H}_{\hat{I}, \hat{P}} \mathbf{s}_{\hat{v}}\right\|_{F}^{2} .
\end{aligned}
$$

Defining the scalar variable $W=\operatorname{tr}\left[-2 \mathcal{R}\left\{\left(\mathbf{H}_{I, P} \mathbf{S}_{v}-\right.\right.\right.$ $\left.\left.\left.\mathbf{H}_{\hat{I}, \hat{P}} \mathbf{S}_{\hat{v}}\right) \mathbf{n}^{H}\right\}\right]$ and trying to find the distribution of $W$ :

$W=\operatorname{tr}\left[-2 \mathcal{R}\left\{\left(\mathbf{H}_{I, P} \mathbf{s}_{v}-\mathbf{H}_{\hat{I}, \hat{P}} \mathbf{s}_{\hat{v}}\right) \mathbf{n}^{H}\right\}\right]$

$$
\begin{aligned}
= & \sum_{l=1}^{2 N_{r}}-2 \mathcal{R}\left\{\left(\mathbf{h}_{l} \mathbf{s}_{v}-\hat{\mathbf{h}}_{l} \mathbf{S}_{\hat{v}}\right) n_{l}\right\}, \quad \mathbf{h}_{l}, \hat{\mathbf{h}}_{l} \text { are the } l \text {-th row of } \mathbf{H}_{I, P} \\
& \text { and } \mathbf{H}_{\hat{I}, \hat{P}} \text { respectively, } \\
= & 2 \sum_{l=1}^{2 N_{r}} \mathcal{I}\left\{\left(\mathbf{h}_{l} \mathbf{s}_{v}-\hat{\mathbf{h}}_{l} \mathbf{s}_{\hat{v}}\right)\right\} \mathcal{I}\left\{n_{l}\right\}-\mathcal{R}\left\{\left(\mathbf{h}_{l} \mathbf{s}_{v}-\hat{\mathbf{h}}_{l} \mathbf{S}_{\hat{v}}\right)\right\} \mathcal{R}\left\{n_{l}\right\}
\end{aligned}
$$

where $\mathcal{R}\left\{n_{l}\right\}$ and $\mathcal{I}\left\{n_{l}\right\}$ are zero-mean independent Gaussian random scalars. As a consequence,

$$
\begin{gathered}
\mathcal{I}\left\{\mathbf{h}_{l} \mathbf{s}_{v}-\hat{\mathbf{h}}_{l} \mathbf{s}_{\hat{v}}\right\} \mathcal{I}\left\{n_{l}\right\} \sim \mathcal{N}\left(0,1 / 2 \sigma^{2} \mathcal{I}\left\{\mathbf{h}_{l} \mathbf{s}_{v}-\hat{\mathbf{h}}_{l} \mathbf{s}_{\hat{v}}\right\}^{2}\right) \\
\mathcal{R}\left\{\mathbf{h}_{l} \mathbf{s}_{v}-\hat{\mathbf{h}}_{l} \mathbf{s}_{\hat{v}}\right\} \mathcal{R}\left\{n_{l}\right\} \sim \mathcal{N}\left(0,1 / 2 \sigma^{2} \mathcal{R}\left\{\mathbf{h}_{l} \mathbf{s}_{v}-\hat{\mathbf{h}}_{l} \mathbf{s}_{\hat{v}}\right\}^{2}\right) .
\end{gathered}
$$

The combination of the previous variables is also zero-mean Gaussian random variable with variance given by $\frac{\sigma^{2}}{2} \mathcal{I}\left\{\mathbf{h}_{l} \mathbf{s}_{v}\right.$ $\left.\hat{\mathbf{h}}_{l} \mathbf{S}_{\hat{v}}\right\}^{2}+\frac{\sigma^{2}}{2} \mathcal{R}\left\{\mathbf{h}_{l} \mathbf{s}_{v}-\hat{\mathbf{h}}_{l} \mathbf{s}_{\hat{v}}\right\}^{2}=\frac{\sigma^{2}}{2}\left\|\mathbf{h}_{l} \mathbf{s}_{v}-\hat{\mathbf{h}}_{l} \mathbf{S}_{\hat{v}}\right\|^{2}$.

Accordingly, the variable $W$ is the sum of zero-mean independent Gaussian random scalars. The variance of $W$ is given by

$$
\begin{aligned}
\operatorname{var}(W) & =4 \sum_{l=1}^{2 N_{r}} \frac{\sigma^{2}}{2}\left\|\mathbf{h}_{l} \mathbf{s}_{v}-\hat{\mathbf{h}}_{l} \mathbf{S}_{\hat{v}}\right\|^{2} \\
& =2 \sigma^{2} \sum_{l=1}^{2 N_{r}}\left(\mathbf{h}_{l} \mathbf{s}_{v}-\hat{\mathbf{h}}_{l} \mathbf{s}_{\hat{v}}\right)\left(\mathbf{h}_{l} \mathbf{s}_{v}-\hat{\mathbf{h}}_{l} \mathbf{s}_{\hat{v}}\right)^{H} \\
& =2 \sigma^{2}\left\|\mathbf{H}_{I, P} \mathbf{s}_{v}-\mathbf{H}_{\hat{I}, \hat{P}} \mathbf{s}_{\hat{v}}\right\|_{F}^{2} .
\end{aligned}
$$

In conclusion, $W=\operatorname{tr}\left[-2 \mathcal{R}\left\{\left(\mathbf{H}_{I, P} \mathbf{S}_{v}-\mathbf{H}_{\hat{I}, \hat{P}} \mathbf{S}_{\hat{v}}\right) \mathbf{n}^{H}\right\}\right]=$ $-2 \mathcal{R}\left\{\mathbf{n}^{H}\left(\mathbf{H}_{I, P} \mathbf{s}_{v}-\mathbf{H}_{\hat{I}, \hat{P}} \mathbf{s}_{\hat{v}}\right)\right\}$ is a zero-mean Gaussian random variable with variance equal to $2 \sigma^{2}\left\|\mathbf{H}_{I, P} \mathbf{S}_{v}-\mathbf{H}_{\hat{I}, \hat{P}} \mathbf{S}_{\hat{v}}\right\|_{F}^{2}$.

\section{APPENDIX B}

The APEP is given by the following double integral

$$
\operatorname{Pr}(\mathbf{x} \rightarrow \hat{\mathbf{x}})=\frac{1}{\pi} \int_{0}^{\pi / 2} \int_{\mathbf{z}} \exp \left(-\frac{\mathbf{z}^{H} \mathbf{z}}{2 \sin ^{2} \theta}\right) f_{\mathbf{z}}(\mathbf{z}) \mathrm{d} \mathbf{z} \mathrm{d} \theta
$$

where $\mathbf{z}$ is assumed to be proper complex Gaussian random vector with joint pdf is given by equation (33). By substituting the pdf of $\mathbf{z}$ in the APEP equation, the second integral is then given by

$A=\int_{\mathbf{z}} \frac{1}{\pi^{2 N r}\left|\boldsymbol{\Sigma}_{\mathbf{z}}\right|} \exp \left(-\frac{\mathbf{z}^{H} \mathbf{z}}{2 \sin ^{2} \theta}-\left(\mathbf{z}-\mathbf{m}_{\mathbf{z}}\right)^{H} \boldsymbol{\Sigma}_{\mathbf{z}}^{-1}\left(\mathbf{z}-\mathbf{m}_{\mathbf{z}}\right)\right) \mathrm{d} \mathbf{z}$

The above equation can be re-expressed by considering the inside of the integral as multivariate scaled Gaussian distribution with mean $\boldsymbol{\mu}_{\mathbf{z}}$ and variance $\boldsymbol{\Sigma}$

$$
-\frac{\mathbf{z}^{H} \mathbf{z}}{2 \sin ^{2} \theta}-\left(\mathbf{z}-\mathbf{m}_{\mathbf{z}}\right)^{H} \boldsymbol{\Sigma}_{\mathbf{z}}^{-1}\left(\mathbf{z}-\mathbf{m}_{\mathbf{z}}\right)=-\left(\mathbf{z}-\boldsymbol{\mu}_{\mathbf{z}}\right)^{H} \boldsymbol{\Sigma}^{-1}\left(\mathbf{z}-\boldsymbol{\mu}_{\mathbf{z}}\right)
$$

+ Const.
The left side of the previous equation can be developed as following

$$
-\mathbf{z}^{H}\left(\boldsymbol{\Sigma}_{\mathbf{z}}^{-1}+\frac{\mathbf{I}}{2 \sin ^{2} \theta}\right) \mathbf{z}+\mathbf{m}_{\mathbf{z}}^{H} \boldsymbol{\Sigma}_{\mathbf{z}}^{-1} \mathbf{z}+\mathbf{z}^{H} \boldsymbol{\Sigma}_{\mathbf{z}}^{-1} \mathbf{m}_{\mathbf{z}}-\mathbf{m}_{\mathbf{z}} \boldsymbol{\Sigma}_{\mathbf{z}}^{-1} \mathbf{m}_{\mathbf{z}}
$$

and the right side can also be developed as

$$
-\mathbf{z}^{H} \boldsymbol{\Sigma}^{-1} \mathbf{z}+\boldsymbol{\mu}_{\mathbf{z}}^{H} \boldsymbol{\Sigma}^{-1} \mathbf{z}+\mathbf{z}^{H} \boldsymbol{\Sigma}^{-1} \boldsymbol{\mu}_{\mathbf{z}}-\boldsymbol{\mu}_{\mathbf{z}}^{H} \boldsymbol{\Sigma}^{-1} \boldsymbol{\mu}_{\mathbf{z}}+\text { Const. }
$$

After identification we can conclude that

$$
\begin{aligned}
\boldsymbol{\Sigma}^{-1} & =\frac{\mathbf{I}}{2 \sin ^{2} \theta}+\boldsymbol{\Sigma}_{z}^{-1} \\
\boldsymbol{\mu}_{\mathbf{z}} & =\boldsymbol{\Sigma} \boldsymbol{\Sigma}_{\mathbf{z}}^{-1} \mathbf{m}_{\mathbf{z}} \\
\text { Const } & =\mathbf{m}_{\mathbf{z}}^{H} \boldsymbol{\Sigma}_{\mathbf{z}}^{-1}\left(\boldsymbol{\Sigma}-\boldsymbol{\Sigma}_{\mathbf{z}}\right) \boldsymbol{\Sigma}_{\mathbf{z}}^{-1} \mathbf{m}_{\mathbf{z}} .
\end{aligned}
$$

As consequence, the new expression of $A$ is given by

$$
\begin{aligned}
A & =\frac{1}{\pi^{2 N r}\left|\boldsymbol{\Sigma}_{\mathbf{z}}\right|} \exp \left(\mathbf{m}_{\mathbf{z}}^{H} \boldsymbol{\Sigma}_{\mathbf{z}}^{-1}\left(\boldsymbol{\Sigma}-\boldsymbol{\Sigma}_{\mathbf{z}}\right) \boldsymbol{\Sigma}_{\mathbf{z}}^{-1} \mathbf{m}_{\mathbf{z}}\right) \times \\
& \int_{\mathbf{z}} \exp \left(-\left(\mathbf{z}-\boldsymbol{\mu}_{\mathbf{z}}\right)^{H} \boldsymbol{\Sigma}^{-1}\left(\mathbf{z}-\boldsymbol{\mu}_{\mathbf{z}}\right)\right) \mathrm{d} \mathbf{z} \\
& =\frac{1}{\left|\boldsymbol{\Sigma}_{\mathbf{z}} \boldsymbol{\Sigma}^{-1}\right|} \exp \left(\mathbf{m}_{\mathbf{z}}^{H} \boldsymbol{\Sigma}_{\mathbf{z}}^{-1}\left(\boldsymbol{\Sigma}-\boldsymbol{\Sigma}_{\mathbf{z}}\right) \boldsymbol{\Sigma}_{\mathbf{z}}^{-1} \mathbf{m}_{\mathbf{z}}\right) \\
& =\frac{1}{\left|\frac{\boldsymbol{\Sigma}_{\mathbf{z}}}{2 \sin ^{2} \theta}+\mathbf{I}\right|} \exp \left(-\mathbf{m}_{\mathbf{z}}^{H}\left(\boldsymbol{\Sigma}_{\mathbf{z}}^{-1}-\boldsymbol{\Sigma}_{\mathbf{z}}^{-1} \mathbf{\Sigma} \boldsymbol{\Sigma}_{\mathbf{z}}^{-1}\right) \mathbf{m}_{\mathbf{z}}\right) .
\end{aligned}
$$

Using the matrix inversion lemma: $\left(A-C B^{-1} D\right)^{-1}=A^{-1}+$ $A^{-1} C\left(B-D A^{-1} C\right)^{-1} D A^{-1}$, we can write that

$$
\left(\boldsymbol{\Sigma}_{\mathbf{z}}^{-1}-\boldsymbol{\Sigma}_{\mathbf{z}}^{-1} \boldsymbol{\Sigma} \boldsymbol{\Sigma}_{\mathbf{z}}^{-1}\right)=\left(\boldsymbol{\Sigma}_{\mathbf{z}}+2 \sin ^{2} \theta \mathbf{I}\right)^{-1} .
$$

Finally, the APEP $\operatorname{Pr}(\mathbf{x} \rightarrow \hat{\mathbf{x}})$ is then given by

$$
\operatorname{Pr}(\mathbf{x} \rightarrow \hat{\mathbf{x}})=\frac{1}{\pi} \int_{0}^{\pi / 2} \frac{\exp \left(-\mathbf{m}_{\mathbf{z}}^{H}\left(\boldsymbol{\Sigma}_{\mathbf{z}}+2 \sin ^{2} \theta \mathbf{I}\right)^{-1} \mathbf{m}_{\mathbf{z}}\right)}{\left|\frac{\boldsymbol{\Sigma}_{\mathbf{z}}}{2 \sin ^{2} \theta}+\mathbf{I}\right|} \mathrm{d} \theta .
$$

\section{APPENDIX C}

More details about the computational complexity of the ML and MOB-MMSE detectors for the DP-GSM system are provided in the following.

\section{A. $M L$ detector}

The ML criterion for DP-GSM can be expressed as follows:

$$
\begin{aligned}
(\hat{I}, \hat{P}, \hat{\mathbf{s}}) & =\underset{I \in \mathbb{I}, P \in \mathbb{P}, \mathbf{s} \in \mathbb{Q}}{\arg \min }\left\|\mathbf{y}-\mathbf{H}_{I, P} \mathbf{s}\right\|^{2} \\
& =\underset{I \in \mathbb{I}}{\arg \min }\left(\min _{P \in \mathbb{P}}\left(\min _{\mathbf{s} \in \mathbb{Q}}\left(\left\|\mathbf{y}-\sum_{k=1}^{N_{a}} \mathbf{h}_{i_{k}} \ell_{k} s_{k}\right\|^{2}\right)\right)\right) .
\end{aligned}
$$

The order of complexity grows as $N_{1} N_{2} M^{N_{a}}=2^{L}$ where $N_{1}=2^{\left\lfloor\log _{2}\left(C_{N_{t}}^{N a}\right)\right\rfloor}$ and $N_{2}=2^{N_{a}}$. For a given $(I, P, \mathbf{s})$, the complexity of the inner term:

$$
\begin{aligned}
& \left\|\mathbf{y}-\sum_{k=1}^{N_{a}} \mathbf{h}_{i_{k} \ell_{k}} s_{k}\right\|^{2}=\sum_{m=1}^{2 N_{r}}\left|y_{m}-\sum_{k=1}^{N_{a}} h_{m, i_{k} \ell_{k}} s_{k}\right|^{2} \\
& =\sum_{m=1}^{2 N_{r}}\left(\mathcal{R}\left(y_{m}-\sum_{k=1}^{N_{a}} h_{m, i_{k} \ell_{k}} s_{k}\right)\right)^{2}+\left(\mathcal{I}\left(y_{m}-\sum_{k=1}^{N_{a}} h_{m, i_{k} \ell_{k}} s_{k}\right)\right)^{2} .
\end{aligned}
$$


Computing $\sum_{k=1}^{N_{a}} h_{m, i_{k}} \ell_{k} s_{k}$ and $\left(\mathcal{R}\left(y_{m}-\sum_{k=1}^{N_{a}} h_{m, i_{k}} \ell_{k} s_{k}\right)\right)^{2}+$ $\left(\mathcal{I}\left(y_{m}-\sum_{k=1}^{N_{a}} h_{m, i_{k}} \ell_{k} s_{k}\right)\right)^{2}$ need $4 N_{a}$ and 2 real-valued multiplications, respectively. As a consequence, $\left\|\mathbf{y}-\sum_{k=1}^{N_{a}} \mathbf{h}_{i_{k}, \ell_{k}} s_{k}\right\|^{2}$ needs $\left(8 N_{a} N_{r}+4 N_{r}\right)$ real-valued multiplications. Hence, the computational complexity of ML detector is $\left(8 N_{a} N_{r}+4 N_{r}\right) 2^{L}$.

\section{B. MOB-MMSE detector}

The computational complexity imposed by the equations given in Algorithm 1 is summarized as follows:

1) $\mathbf{h}_{i_{q} \ell_{q}}^{H} \mathbf{y}$ takes $8 N_{r}$ real-valued multiplications.

2) $\left.\left|\mathbf{h}_{i_{q} \ell_{q}}^{H} \mathbf{y}\right|^{2}=\mathcal{R}\left(\mathbf{h}_{i_{q} \ell_{q}}^{H} \mathbf{y}\right)\right)^{2}+\mathcal{I}\left(\mathbf{h}_{i_{q} \ell_{q}}^{H} \mathbf{y}\right)^{2}$ takes $8 N_{r}+2$ realvalued multiplications.

3) $\mathbf{h}_{i_{q} \ell_{q}}^{H} \mathbf{h}_{i_{q} \ell_{q}}=\sum_{j=1}^{2 N_{r}}\left|h_{j, i_{q} \ell_{q}}\right|^{2}=\sum_{j=1}^{2 N_{r}} \mathcal{R}\left(h_{j, i_{q} \ell_{q}}\right)^{2}+\mathcal{I}\left(h_{j, i_{q} \ell_{q}}\right)^{2}$ takes $4 N_{r}$ real-valued multiplications.

4) $\left|z_{i_{q} \ell_{q}}\right|^{2}=\frac{\left|\mathbf{h}_{i_{q} \ell_{q}}^{H} \mathbf{y}\right|^{2}}{\left(\mathbf{h}_{i_{q} \ell_{q}}^{H} \mathbf{h}_{i_{q} \ell_{q}}\right)^{2}}$ takes $12 N_{r}+4$ real-valued multiplications. As a consequence, $\mathbf{z}$ takes $\left(12 N_{r}+4\right) 2 N_{t}$ realvalued multiplications.

5) $w^{i, \ell}=\sum_{q=1}^{N_{a}}\left|z_{i_{q}} \ell_{q}\right|^{2}$ doesn't take any multiplications.

6) $\mathbf{H}_{I_{i \leftarrow k_{n}}^{H}, P_{\ell \leftarrow k n}}^{H} \mathbf{y}$ takes $8 N_{r} N_{a}$ real-valued multiplications.

7) $\mathbf{H}_{I_{i \leftarrow k_{n}}^{H}, P_{\ell \leftarrow k_{n}}^{H}}^{H} \mathbf{H}_{I_{i \leftarrow k_{n}}, P_{\ell \leftarrow k_{n}}}$ takes $8 N_{r} N_{a}^{2}$ real-valued multiplications.

8) Since $\mathbf{R}=\mathbf{H}_{I_{i \leftarrow k_{n}}^{H}, P_{\ell \leftarrow k_{n}}} \mathbf{H}_{I_{i \leftarrow k_{n}}, P_{\ell \leftarrow k_{n}}}+\sigma^{2} \mathbf{I}_{N_{a}}$ is complex Hermitian matrix positive-definite then $\mathbf{R}^{-1}$ takes $2 N_{a}^{3}+6 N_{a}^{2}$ real-valued multiplications [42]. Hence, $\left(\mathbf{H}_{I_{i \leftarrow k_{n}}, P_{\ell \leftarrow k_{n}}}^{H} \mathbf{H}_{I_{i \leftarrow k n}, P_{\ell \leftarrow k n}}+\sigma^{2} \mathbf{I}_{N_{a}}\right)^{-1}$ takes $8 N_{r} N_{a}^{2}+$ $2 N_{a}^{3}+6 N_{a}^{2}$.

9) $\left(\mathbf{H}_{I_{i \leftarrow k n}}^{H}, P_{\ell \leftarrow k n} \mathbf{H}_{I_{i \leftarrow k n}, P_{\ell \leftarrow k n}}+\sigma^{2} \mathbf{I}\right)^{-1} \mathbf{H}_{I_{i \leftarrow k_{n}}, P_{\ell \leftarrow k n}}^{H} \mathbf{y}$ takes $8 N_{r} N_{a}^{2}+2 N_{a}^{3}+6 N_{a}^{2}+8 N_{r} N_{a}+4 N_{a}^{2}$ real-valued multiplications.

10) The digital demodulator function $\mathcal{D}$ takes $2 M N_{a}$ realvalued multiplications to detect the $N_{a}$ symbols.

11) $d_{n}=\left\|\mathbf{y}-\mathbf{H}_{I_{i \leftarrow k_{n}}, P_{\ell \leftarrow k_{n}}} \tilde{\mathbf{s}}_{n}\right\|^{2}$ takes $8 N_{r} N_{a}+4 N_{r}$ realvalued multiplications

12) For a each iteration $n$ of the loop, the detection process needs $8 N_{r} N_{a}^{2}+\left(2 N_{a}^{3}+6 N_{a}^{2}\right)+8 N_{r} N_{a}+4 N_{a}^{2}+\left(8 N_{r} N_{a}+\right.$ $\left.4 N_{r}\right)+2 M N_{a}$ real-valued multiplications.

13) For all the process (while $n \leq N$ do), we define $\bar{N} \leq$ $N$ the average number of iteration executed in MOBMMSE algorithm to detect the TAPC and APM symbols. Then, the computational complexity of the loop is $\left[2 N_{a}^{3}+\right.$ $\left.N_{a}^{2}\left(8 N_{r}+10\right)+N_{a}\left(16 N_{r}+2 M\right)+4 N_{r}\right] \bar{N}$.

14) Finally, the total computational complexity of the MOBMMSE without any pre-computation is:

$$
\begin{aligned}
& \left(2 N_{a}^{3}+N_{a}^{2}\left(8 N_{r}+10\right)+N_{a}\left(16 N_{r}+2 M\right)+4 N_{r}\right) \bar{N} \\
& +24 N_{r} N_{t}+8 N_{t} .
\end{aligned}
$$

\section{ACKNOWLEDGMENT}

The research leading to these results received funding from the French National Research Agency (ANR-17-ce25-0013) within the frame of the project BRAVE (Back to single-CaRrier for beyond-5G communications AboVE 90GHz). We would like to thank BRAVE project partner SIRADEL for providing the sub-THz MIMO channels from their ray-based channel simulation.

\section{REFERENCES}

[1] S. Sugiura, S. Chen, and L. Hanzo, "A universal space-time architecture for multiple-antenna aided systems," IEEE Commun. Surv. Tuts., vol. 14,no. 2, pp. 401-420, Jan. 2012.

[2] L. Zheng and D. N. C. Tse, "Diversity and multiplexing: A fundamental trade-off in multiple-antenna channels," IEEE Trans. Inf. Theory, vol. 49,no. 5, pp. 1073-1096, May 2003.

[3] M. Saad, F. Bader, J. Palicot, Y. Corre, G. Gougeon, J-B Dore, "Beyond$5 \mathrm{G}$ wireless Tbps Scenarios and Requirements," French funded projectANR-17-CE25-0013 BRAVE, Tech. Report BRAVE D1.0, 2018. [Online]. Available: https://hal.archives-ouvertes.fr/hal-01947363/document.

[4] Matti Latva-aho, Kari Leppänen, 6G Research Visions 1, "Key Drivers and Research Challenges for 6G Ubiquitous Wireless Intelligence", $6 G$ Summit Conference, Oulu, Finland, September 2019.

[5] S. Bicaïs and J.-B. Doré, "Phase Noise Model Selection for Sub-THz Communications," in the Proc. of the IEEE Global Communication Conference (Globecom'19), Waikoloa, HI, USA. Dec 2019.

[6] M. Saad, A. C. Al Ghouwayel, H. Hijazi, F. Bader and J. Palicot,"MIMO Techniques for Wireless Terabits Systems under sub-THz Channel with RF Impairments," in IEEE International Conference on Communications-ICC, Dublin, Ireland, 2020, pp. 1-6..

[7] French funded project-ANR-17-CE25-0013, "Back to Single-carrier for beyond-5G communications above $90 \mathrm{GHz}$-(BRAVE)," [Online]. Available: http://www.brave-beyond5g.com/.

[8] J.-B. Dore, Y. Corre, S. Bicais, J. Palicot, E. Faussurier, D. Ktenas, and F. Bader, "Above-90GHz Spectrum and Single-Carrier Waveform as Enablers for Efficient Tbit/s Wireless Communications," IEEE 25th International Conference on Telecommunications (ICT), Saint-Malo, France, 2018.

[9] P. Yang, M. Di Renzo, Y. Xiao, S. Li, and L. Hanzo, "Design guide-lines for spatial modulation," IEEE Commun. Surv. Tuts., vol. 17, no. 1,pp. 6-26, May 2014.

[10] J. T. Wang, S. Y. Jia, J. Song, "Generalised spatial modulation system with multiple active transmit antennas and low complexity detection scheme," IEEE Trans. Wireless Commun., vol.11, no. 4, pp. 1605-1615, Apr. 2012.

[11] M. Di Renzo, H. Haas, A. Ghrayeb, S. Sugiura, and L. Hanzo, "Spatial modulation for generalized MIMO: Challenges, opportunities, and implementation," Proc. IEEE, vol. 102, no. 1, pp. 56-103, Jan. 2014.

[12] M. Saad, F. Bader, J. Palicot, A. C. A. Ghouwayel and H. Hijazi, "Single Carrier with Index Modulation for Low Power Terabit Systems," 2019 IEEE Wireless Communications and Networking Conference (WCNC), Marrakesh, Morocco, 2019, pp. 1-7.

[13] M. Saad, F. C. Lteif, A. C. Al Ghouwayel, H. Hijazi, J. Palicot and F. Bader, "Generalized Spatial Modulation in Highly Correlated Channels," 2019 IEEE 30th International Symposium on Personal, Indoor and Mobile Radio Communications (PIMRC Workshops), Istanbul, Turkey, 2019, pp. 1-6.

[14] G. Zafari, M. Koca and H. Sari, "Dual-Polarized Spatial Modulation Over Correlated Fading Channels," in IEEE Trans. Commun., vol. 65, no. 3, pp. 1336-1352, March 2017.

[15] Y. Xiao, Z. Yang, L. Dan, P. Yang, L. Yin and W. Xiang, "LowComplexity Signal Detection for Generalized Spatial Modulation," in IEEE Commun. Lett., vol. 18, no. 3, pp. 403-406, March 2014.

[16] F. Wang, Y. Xiong and X. Yang. "Approximate ML Detection Based on MMSE for MIMO Systems.” Piers Online, Vo. 3, pp.475-480. 2007.

[17] S. L. Loyka, "Channel capacity of MIMO architecture using the exponential correlation matrix," in IEEE Commun. Lett., vol. 5, pp.369-371, Sept. 2001.

[18] K. P. Liolis, J. Gomez-Vilardebo, E. Casini and A. I. Perez-Neira, "Statistical Modeling of Dual-Polarized MIMO Land Mobile Satellite Channels," in IEEE Trans. Commun., vol. 58, no. 11, pp. 3077-3083, November 2010.

[19] C. Oestges, B. Clerckx, M. Guillaud and M. Debbah, "Dual-polarized wireless communications: from propagation models to system performance evaluation," in IEEE Trans. Wireless Commun., vol. 7, no. 10, pp. 4019-4031, October 2008. 
[20] H. Asplund, J. Berg, F. Harrysson, J. Medbo and M. Riback, "Propagation Characteristics of Polarized Radio Waves in Cellular Communications," 2007 IEEE 66th Vehicular Technology Conference, Baltimore, MD, 2007, pp. 839-843.

[21] M. Saad, F. Bader, A. C. Al Ghouwayel, H. Hijazi, N. Bouhel and J. Palicot, "Generalized Spatial Modulation for Wireless Terabits Systems Under Sub-THZ Channel With RF Impairments," ICASSP 2020 2020 IEEE International Conference on Acoustics, Speech and Signal Processing (ICASSP), Barcelona, Spain, 2020, pp. 5135-5139.

[22] N. Bouhlel, M. Saad, F. Bader, and J. Palicot,"Dual-Polarized Generalized Spatial Modulation for Wireless Terabit System," in 2020 IEEE 31st Annual International Symposium on Personal, Indoor and Mobile Radio Communications (PIMRC), London, UK, 2020, pp. 1-7

[23] H. Sarieddeen, M-S Alouini, T. Y. Al-Naffouri, "An Overview of Signal Processing Techniques for Terahertz Communications," arXiv preprint arXiv:2005.13176, 2020,

[24] G. Gougeon, Y. Corre and M. Z. Aslam, "Ray-based Deterministic Channel Modelling for sub-THz Band," 2019 IEEE 30th International Symposium on Personal, Indoor and Mobile Radio Communications (PIMRC Workshops), Istanbul, Turkey, 2019, pp. 1-6.

[25] A. Chatterjee, S. Chatterjee and S. S. Das, "Evaluation of spatial correlation and its effect on channel capacity of uniform planar antenna array," 2017 Twenty-third National Conference on Communications (NCC), Chennai, 2017, pp. 1-6.

[26] B. Murmann, "ADC Performance Survey 1997-2019," [Online]. Available: http://web.stanford.edu/ murmann/adcsurvey.html

[27] F. D. Neeser and J. L. Massey, "Proper complex random processes with applications to information theory," in IEEE Trans. Inf. Theory, vol. 39 no. 4, pp. 1293-1302, July 1993.

[28] V. V. Veeravalli, "On performance analysis for signaling on correlated fading channels," in IEEE Trans. Commun., vol. 49, no. 11, pp. 18791883, Nov. 2001,

[29] K. Brijesh and K. Rakhesh Singh, "MIMO Wireless Communications over Generalized Fading Channels (1st. ed.)". CRC Press, Inc., USA, 2017.

[30] G. Proakis, Digital Communications, 4th ed. New York, NY, USA: McGraw-Hill, 2000.

[31] K. Ishibashi and S. Sugiura, "Effects of Antenna Switching on BandLimited Spatial Modulation," in IEEE Wireless Commun. Lett., vol. 3 , no. 4, pp. 345-348, Aug. 2014.

[32] E. Soujeri and G. Kaddoum, "The Impact of Antenna Switching Time on Spatial Modulation," in IEEE Wireless Commun. Lett., vol. 5, no. 3, pp. 256-259, June 2016.

[33] M. Nakao, T. Ishihara, and S. Sugiura, "Single-carrier frequency domain equalization with index modulation," IEEE Commun. Lett., vol. 21, no. 2, pp. 298-301, Feb. 2017.

[34] D. Tsonev, S. Sinanovic, and H. Haas, "Enhanced subcarrier index modulation (SIM) OFDM," in Proc. IEEE GLOBECOM Workshops, Dec. 2011, pp. 728-732.

[35] E. Basar, U. Aygolu, E. Panayirci, and H. V. Poor, "Orthogonal frequency division multiplexing with index modulation," in IEEE Trans. Signal Process., vol. 61, no. 22, pp. 5536-5549, 2013.

[36] M. Chafii, F. Bader and J. Palicot, "SC-FDMA with index modulation for M2M and IoT uplink applications," in 2018 IEEE Wireless Commun. and Netw. Conf. (WCNC), Barcelona, 2018, pp. 1-5.

[37] J. Jeganathan, A. Ghrayeb, L. Szczecinski and A. Ceron, "Space shift keying modulation for MIMO channels," in IEEE Trans. Wireless Commun., vol. 8, no. 7, pp. 3692-3703, July 2009.

[38] L. Yang, "Transmitter Preprocessing Aided Spatial Modulation for Multiple-Input Multiple-Output Systems," in 2011 IEEE 73rd Veh. Technol. Conf. (VTC Spring), Yokohama, 2011, pp. 1-5.

[39] P. Liu, M. Di Renzo and A. Springer, "Variable- $N_{u}$ generalized spatial modulation for indoor LOS mmWave communication: Performance optimization and novel switching structure," in IEEE Trans. Commun., vol. 65 , no. 6, pp. 2625-2640, June 2017.

[40] M. Saad, J. Palicot, F. Bader, A. C. Al Ghouwayel and H. Hijazi, "A Novel Index Modulation Dimension based on Filter Domain: Filter Shapes Index Modulation," in IEEE Trans. Commun., vol. 69, no. 3, 2021. doi:10.1109/TCOMM.2020.3039842.

[41] M. Saad, N. Al Akkad, H. Hijazi, A. Chamas Al Ghouwayel, F. Bader and J. Palicot, "Novel MIMO Technique for Wireless Terabits Systems in sub-THz Band," in IEEE Open Journal of Veh. Technol., vol. 2, 2021. doi: 10.1109/OJVT.2021.3054737.

[42] R. Hunger, "Floating point operations in matrix-vector calculus," Technische Universitat Munchen, Associate Institute for Signal Processing, Tech. Rep., 2007.

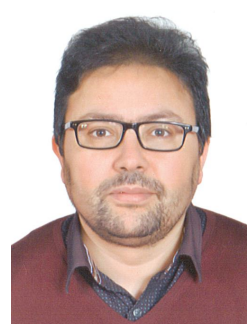

Nizar Bouhlel received the engineering degree in electrical engineering and master degree in communication systems from the Ecole Nationale d'Ingénieurs de Tunis (ENIT), Tunisia, in 1997 and 1999, respectively. He received the Ph.D. degree in Applied Mathematics from the University of Paris Descartes, MAP5, France, in 2006. He worked from 2007 until 2011 as a researcher at the Faculty of Sciences of Monastir, Communication department. From 2012 until 2020, he was post-doctoral researcher in several French laboratories: Laboratoire d'Imagerie Biomédicale (LIB) at UPMC, Laboratoire des Sciences du Numérique (LS2N) at ECN, Institute of Electronics and Telecommunications of Rennes (IETR) with the team SHINE, INSA Rennes and IETR at CentraleSupélec, Rennes, France. His current research focuses on stochastic signal and image processing, radar signal processing and signal processing for digital communications. In December 2020, he was hired as assistant professor at the Institut Agro, Statistics and Computer Science Department, Angers, France and in the Research Institute on Horticulture and Seeds (IRHS), team "Imagerie pour l'Horticulture et le Phénotypage" (ImHorPhen). His current research focuses on stochastic signal and image processing, radar signal processing and signal processing for digital communications.

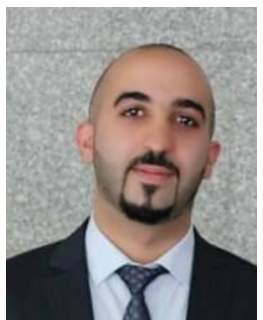

Majed Saad (Member, IEEE) received the M. Sc. degree with highest distinction in computer and communication engineering (CCE) from Lebanese International University (LIU), Beirut, Lebanon, in 2016 and the Ph.D. degree in signal processing and telecommunications from CentraleSupélec, Rennes, France, in 2020. He secured the first rank during his M.Sc. studies with the CCE Department on all LIU campuses, Lebanon. From October 2016 to February 2020 , he was also a Lab Instructor with the School of Engineering, LIU. Since January 2018, he has been involved with the BRAVE French National Project (ANR) about beyond 5G terabits wireless communication in the sub-terahertz bands. Since September 2020, he has been a Postdoctoral Researcher with CentraleSupélec, France. His main research interests include beyond 5G, millimeter wave, sub- $\mathrm{THz}$ and terahertz systems, MIMO systems, index modulation, signal processing, and equalization. He was the recipient of the grant from the CNRS GdR-ISIS in France for mobility and cooperation with the University of Patras, Greece, in 2019. He was also a Reviewer for several IEEE conferences and the IEEE TRANSACTIONS ON COMMUNICATIONS.

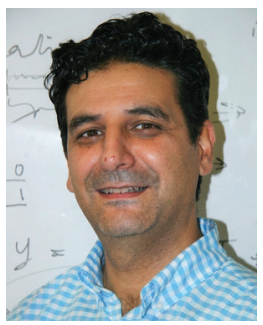

Faouzi Bader (M'02-SM'07) received the Ph.D. degree (Hons.) in telecommunications from the Universidad Politécnica de Madrid, Madrid, Spain, in 2002. He joined the Centre Technològic de Telecomunicacion de Catalunya-CTTC, Barcelona, Spain, as Research Associate in 2002, where he has been a Senior Research Associate from 2006 to 2013. Since June 2013, he has been Associate Professor with CentraleSupélec, France. His research activities mainly focus on IMT-advanced systems as $\mathrm{THz}$ wireless communications, $5 \mathrm{G}$ networks and systems, and cognitive radio communication environment. He has been involved in several European projects from the 5th-7th EC research frameworks (eight EU funded projects and ten national projects), from 2012 to 2013 he has been the General Coordinator and Manager of the EC funded ICT "EMPhAtiC" project focusing on "Enhanced Multicarrier Techniques for Professional Ad-Hoc and Cell-Based Communications". Since 2017, he is Honorary Adjunct Professor with University Technology Sydney, Australia. From 2018-2020, he has been as the Head of the Signals and Communications Department of Institute of Electronics and Telecommunications of Rennes (IETR), France. Since 2020, he is the director of research at the Institut Supérieur d'Électronique de Paris-ISEP (France). He published over 32 journals and 128 papers in peer-reviewed international conferences, over 13 book chapters, and 5 edited books. He served as the General Chair, the Technical Program Co-Chair, and the Technical Co-Chair of symposia at numerous major IEEE ComSoc and VTS conferences, such as IEEE ICC, IEEEPIMRC, IEEE VTC spring/fall, IEEE WCNC, ISWCS, IEEE GLOBECOM, and ICT. 